\title{
Resolved magnetic structures in the disk-halo interface of NGC 628 ^
}

\author{
D. D. Mulcahy ${ }^{1, \star \star}$, R. Beck ${ }^{2}$, and G. H. Heald ${ }^{3,4,5}$ \\ 1 Jodrell Bank Centre for Astrophysics, Alan Turing Building, School of Physics and Astronomy, The University of Manchester, \\ Oxford Road, Manchester, M13 9PL, UK \\ e-mail: david.mulcahy@manchester.ac.uk \\ 2 Max-Planck-Institut für Radioastronomie, Auf dem Hügel 69, 53121 Bonn, Germany \\ 3 CSIRO Astronomy and Space Science, 26 Dick Perry Avenue, Kensington, WA 6151, Australia \\ ${ }^{4}$ Netherlands Institute for Radio Astronomy (ASTRON), Postbus 2, 7990 AA Dwingeloo, The Netherlands \\ 5 Kapteyn Astronomical Institute, Postbus 800, 9700 AV Groningen, The Netherlands
}

Received 14 October 2016/ Accepted 20 December 2016

\begin{abstract}
Context. Magnetic fields are essential to fully understand the interstellar medium and its role in the disk-halo interface of galaxies is still poorly understood. Star formation is known to expel hot gas vertically into the halo and these outflows have important consequences for mean-field dynamo theory in that they can be efficient in removing magnetic helicity.

Aims. We aim to probe the vertical magnetic field and enhance our understanding of the disk-halo interaction of galaxies. Studying a face-on galaxy is essential so that the magnetic field components can be separated in 3D.

Methods. We perform new observations of the nearby face-on spiral galaxy NGC 628 with the Karl G. Jansky Very Large Array (JVLA) at $S$-band (2.6-3.6 GHz effective bandwidth) and the Effelsberg $100-\mathrm{m}$ telescope at frequencies of $2.6 \mathrm{GHz}$ and $8.35 \mathrm{GHz}$ with a bandwidth of $80 \mathrm{MHz}$ and $1.1 \mathrm{GHz}$, respectively. Owing to the large bandwidth of the JVLA receiving system, we obtain some of the most sensitive radio continuum images in both total and linearly polarised intensity of any external galaxy observed so far.

Results. The application of rotation measures synthesis to the interferometric polarisation data over this large bandwidth provides high-quality images of Faraday depth and polarisation angle from which we obtained evidence for drivers of magnetic turbulence in the disk-halo connection. Such drivers include a superbubble detected via a significant Faraday depth gradient coinciding with a $\mathrm{H}_{\mathrm{I}}$ hole. We observe an azimuthal periodic pattern in Faraday depth with a pattern wavelength of $3.7 \pm 0.1 \mathrm{kpc}$, indicating Parker instabilities. The lack of a significant anti-correlation between Faraday depth and magnetic pitch angle indicates that these loops are vertical in nature with little helical twisting, unlike in IC 342. We find that the magnetic pitch angle is systematically larger than the morphological pitch angle of the polarisation arms which gives evidence for the action of a large-scale dynamo where the regular magnetic field is not coupled to the gas flow and obtains a significant radial component. We additionally discover a lone region of ordered magnetic field to the north of the galaxy with a high degree of polarisation and a small pitch angle, a feature that has not been observed in any other galaxy so far and is possibly caused by an asymmetric $\mathrm{H}_{\mathrm{I}}$ hole.

Conclusions. Until now NGC 628 has been relatively unexplored in radio continuum but with its extended H i disk and lack of active star formation in its central region has produced a wealth of interesting magnetic phenomena. We observe evidence for two drivers of magnetic turbulence in the disk-halo connection of NGC 628, namely, Parker instabilities and superbubbles.
\end{abstract}

Key words. cosmic rays - galaxies: magnetic fields - galaxies: ISM - radio continuum: galaxies

\section{Introduction}

Understanding of the disk-halo interaction is vital to explain the evolution of spiral galaxies. Several theories exist that describe how star formation expels hot gas vertically into the halo, namely the galactic fountain model (Shapiro \& Field 1976; Bregman 1980), the chimney model (Norman \& Ikeuchi 1988), and the galactic wind model (Breitschwerdt et al. 1991) relevant to quiescent star formation (SF), clustered SF and starbursts respectively.

\footnotetext{
* The reduced images/datacubes (FITS files) are only available at the CDS via anonymous ftp to cdsarc.u-strasbg.fr (130.79.128.5) or via http://cdsarc.u-strasbg.fr/viz-bin/qcat?J/A+A/600/A6 $\star \star$ Based on observations with the Karl G. Jansky JVLA of the NRAO at Socorro and the 100-m telescope of the Max-Planck-Institut für Radioastronomie at Effelsberg.
}

These disk-halo outflows have important consequences for mean-field dynamo theory, a process which involves the inductive effect of turbulence and differential rotation to amplify the weak magnetic fields to produce strong large-scale fields (Ruzmaikin et al. 1988; Beck et al. 1996). Brandenburg et al. (1995) studied a simple model of such a galactic fountain flow and found that the horizontal field in the galactic disk can be pumped out into the halo to a height of several kpcs. The magnetic field strength at a height of several kpcs was found to be comparable to that in the disk. Mean-field dynamo models including a galactic wind may also explain the X-shaped magnetic fields observed around edge-on galaxies (Moss et al. 2010). Alternatively, suppression of the $\alpha$-effect and of mean-field dynamo action can result from the conservation of magnetic helicity in a medium of high electric conductivity. Shukurov et al. (2006) showed that the galactic fountain flow is efficient in removing magnetic helicity from galactic disks. This allows the 
mean magnetic field to saturate at a strength comparable to equipartition with the turbulent kinetic energy. However, fast outflows can advect large-scale magnetic fields and hence suppress mean-field dynamo action. As the outflow is expected to be stronger above and below the spiral arms, large-scale fields are weaker than in the inter-arm regions which may explain the observation of magnetic arms between material arms (Chamandy et al. 2015).

Such outflows driven from stellar winds and supernovae from young massive stars in $\mathrm{OB}$ associations and super star clusters (SSCs) create Hi holes (Bagetakos et al. 2011) and magnetic field loops pushed up by the gas (Bregman 1980; Norman \& Ikeuchi 1988). As a result, gradients in Faraday rotation measures (RM) and field reversals across these $\mathrm{H}_{\mathrm{I}}$ holes are expected. Detecting such RM gradients would constrain the parameters of this outflow model.

Magnetic fields oriented along the disk plane may also bend upwards and become vertical fields as the result of the magnetic Rayleigh-Taylor instability, commonly known as the Parker instability. Ionised gas can slide down the magnetic loops, thereby reducing the confining weight of the gas and allowing the loops to rise even higher.

Efforts have been made to observe magnetic field loops. Heald (2012) detected an RM gradient in NGC 6946 across a $600-\mathrm{pc} \mathrm{H}_{\mathrm{I}}$ hole which indicated a vertical magnetic field. However, this observational result was obtained for an inclined galaxy, so that the observed RM fluctuations could be affected by the variations of field strengths either parallel or vertical to the disk. Field amplification in the disk can be due to compressive gas motions (density waves) or shearing, while vertical fluctuations can be due to Parker loops, which are of kpc scale, or gas outflows. A helically twisted Parker loop was recently found in the spiral galaxy IC 342 (Beck 2015).

Studying a face-on galaxy like NGC 628 is essential so that the magnetic field components can be separated in $3 \mathrm{D}$. In this case, RMs are only sensitive to vertical fields, while synchrotron emission traces the magnetic field in the disk.

NGC $628=$ M74 is a large, grand-design, isolated spiral galaxy with a low inclination angle and a $\mathrm{H}$ i disk with a diameter of about 30', about three times the Holmberg diameter (Kamphuis \& Briggs 1992). A list of physical parameters is given in Table 1. The spiral structure is grand-design but with broad spiral arms. The apparent absence of strong density waves is related to the lack of a strong bar or an interacting companion, leaving the disk largely undisturbed (seen in optical, UV, and in gas kinematics). NGC 628 is similar to NGC 6946 in many respects and hosts many $\mathrm{H}_{\mathrm{I}}$ holes (Bagetakos et al. 2011). NGC 628 actually contains nearly twice the number of H I holes (102 holes) compared to NGC 6946 (54 holes) found by Bagetakos et al. (2011), but previous studies performed by Boomsma et al. (2008) found $121 \mathrm{H}$ i holes for NGC 6946 with inferior resolution compared to Bagetakos et al. (2011).

The high star-formation rate (SFR) of NGC 628 leads to a bright disk in radio continuum (synchrotron + thermal) of about $10^{\prime} \times 8^{\prime}$ extent (Condon 1987). Marcum et al. (2001) observed NGC 628 extensively in UV. Bright knots embedded in diffuse emission trace the spiral pattern and many of these knots are also bright in $\mathrm{H} \alpha$ (Dale et al. 2009). Marcum et al. (2001) suggested that the entire disk of NGC 628 has undergone active star formation within the past $500 \mathrm{Myr}$ and that the inner regions have experienced more rapidly declining star formation than the outer regions.

NGC 628 was part of the Westerbork Synthesis Radio Telescope (WSRT) SIRTF Nearby Galaxies Survey (SINGS)
Table 1. Physical parameters of NGC 628.

\begin{tabular}{|c|c|}
\hline Morphology ${ }^{a}$ & SAc \\
\hline Position of the nucleus & $\begin{aligned} \operatorname{RA}(J 2000) & =01^{\mathrm{h}} 36^{\mathrm{m}} 41^{\mathrm{s}} 74 \\
\operatorname{Dec}(\mathrm{J} 2000) & =+15^{\circ} 47^{\prime} 01.1^{\prime \prime}\end{aligned}$ \\
\hline Position angle of major axis ${ }^{b}$ & $20^{\circ}$ \\
\hline $\mathrm{D}_{25}{ }^{a}$ & $10.5 \times 9.5^{\prime}$ \\
\hline Inclination of the inner $\operatorname{disk}^{b}$ & $7^{\circ}\left(0^{\circ}\right.$ is face on $)$ \\
\hline Inclination of the outer $\operatorname{disk}^{b}$ & $13.5^{\circ}$ \\
\hline Distance $^{c}$ & $7.3 \mathrm{Mpc}\left(1^{\prime \prime} \approx 35 \mathrm{pc}\right)$ \\
\hline Star formation rate ${ }^{d}$ & $1.21 M_{\odot} \mathrm{yr}^{-1}$ \\
\hline
\end{tabular}

References. ${ }^{(a)}$ de Vaucouleurs et al. (1991); (b) Kamphuis \& Briggs (1992); ${ }^{(c)}$ Karachentsev et al. (2004); ${ }^{(d)}$ Walter et al. (2008).

survey and was also observed in polarised radio continuum at 1.5 GHz with moderate resolution (Heald et al. 2009). The polarised emission is restricted to the outer disk and the observed Faraday depths are small, due to strong Faraday depolarisation at this frequency, similar to NGC 6946 observed at the same frequency. With the exception of this observation, NGC 628 has not been studied in radio continuum.

Due to its small inclination and large angular size, NGC 628 is one of the best galaxies to study the magnetic field of the disk. However, its low inclination makes it difficult to extract an accurate rotation curve and was not computed in the THINGS H i Nearby Galaxy Survey (de Blok et al. 2008). Kamphuis \& Briggs (1992) derived from their H I observations that the rotation velocity reaches $200 \mathrm{~km} \mathrm{~s}^{-1}$.

In this paper, we present new Karl G. Jansky Very Large Array (JVLA) $S$-band observations of NGC 628 along with Effelsberg observations at $2.6 \mathrm{GHz}$ and $8.35 \mathrm{GHz}$ including both total power and polarisation. Section 2 presents details of the observations and data reduction, Sect. 3 shows the Effelsberg maps and comparison between the extracted flux densities with literature. In Sect. 4 we present the new JVLA observations, compare to observations at other wavelengths, and determine magnetic field strengths. In Sect. 5 we show results of RM synthesis in probing the vertical magnetic field of NGC 628. In Sect. 6 we discuss the implications of our main findings and in Sect. 7 we summarise our findings.

\section{Observations and data reduction}

In this section we describe the nature of the observations taken with the JVLA and Effelsberg 100-m telescope and the steps performed in the data reduction, starting with the Effelsberg observations. A brief summary of the observational parameters is given in Table 2 .

\subsection{Effelsberg observations at $2.6 \mathrm{GHz}$ and $8.35 \mathrm{GHz}$}

NGC 628 was observed at $2.6 \mathrm{GHz}$ and $8.35 \mathrm{GHz}$ (with bandwidths of $80 \mathrm{MHz}$ and $1.1 \mathrm{GHz}$, respectively) in October 2011 by the authors. The maps were scanned alternating in RA and Dec with the one-horn secondary-focus systems. The data was reduced with the NOD2 software (Haslam 1974). As radio frequency interference (RFI) can be a substantial problem at $8.35 \mathrm{GHz}$, NGC 628 was only observed at higher elevations at this frequency. 4 out of 32 coverages had to be discarded due to extremely bad RFI. NGC 628 was observed at $2.6 \mathrm{GHz}$ at lower elevations as the RFI is not as severe at this frequency. However, significant RFI was still present and had to be flagged in 
Table 2. Radio continuum observational parameters of NGC 628.

\begin{tabular}{lcc}
\hline \hline & JVLA & Effelsberg \\
\hline Frequency $(\mathrm{GHz})$ & $2-4$ & 2.6 and 8.35 \\
Bandwidth (MHz) & 2000 (of which 1000 are useful) & 80 and 1100 \\
No. of spectral windows & 16 & - \\
Total no. of channels & 1024 & 8 and 1 \\
Array configuration & D; C & - \\
Pointings & 7 & - \\
Observing dates & March \& July 2013, respectively & October 2011 \\
\hline
\end{tabular}

Stokes $I, Q$, and $U$. The final Effelsberg images at $2.6 \mathrm{GHz}$ and $8.35 \mathrm{GHz}$ were obtained from 13 and 28 coverages, respectively, and combined using the spatial-frequency weighting method of Emerson \& Gräve (1988).

A flux density scaling was applied to the $8.35 \mathrm{GHz}$ data using maps of two calibrators, 3C 286 and 3C 48. Using values from the VLA calibrator handbook ${ }^{1}$, a common scale factor was found and was applied to the target data. For both calibrators, the linearly polarised intensity and polarisation angle were found by fitting a 2D-Gaussian over the Stokes $Q$ and $U$ maps and calculated from the following equations:

$P_{\text {lin }}=\sqrt{U^{2}+Q^{2}-\left(1.2 \sigma_{Q U}\right)^{2}}$

where $\sigma$ is the noise in Stokes $Q$ and $U$,

$\phi=\frac{1}{2} \arctan \left(\frac{U}{Q}\right)$

From the seven maps of $3 \mathrm{C} 48$, one had a very different value for the polarisation angle and was discarded. The remaining maps gave a degree of polarisation of $5.5 \%$ and a polarisation angle of $-65^{\circ}$. This agrees very well with the values of $5.3 \%$ and $-64^{\circ}$ for $8.1 \mathrm{GHz}$ found by Perley \& Butler (2013b). In addition, for two maps of $3 \mathrm{C} 286$, the degree of polarisation was $11.65 \%$ and the polarisation angle was $33.8^{\circ}$. Again, this is in excellent agreement to Perley \& Butler (2013b) who found values of $11.7 \%$ and $33^{\circ}$. Therefore, we can be confident that the polarisation calibration for our target is accurate. The level of instrumental polarisation is below $1 \%$ at both frequencies.

As the $2.6 \mathrm{GHz}$ receiver is equipped with a 8 channel polarimeter, an additional bandwidth calibration had to be performed along with the normal flux density scaling. For each of the eight channels, the flux density of the calibrator 3C 295 was compared to its theoretical value, using a power law with a spectral index of -1.04 . A scale factor was then determined from the ratio of the two and applied to each channel. The final images for both frequencies are presented in Figs. 1 and 2.

\subsection{JVLA observations at S-band}

NGC 628 was observed by the JVLA in D and C configurations in February and June 2013 in $S$-band (2-4 GHz). The synthesised beamwidth for a $12 \mathrm{~h}$ observation with uniform weighting scheme is approximately $23^{\prime \prime}$ for D configuration at $3 \mathrm{GHz}$ and $7.0^{\prime \prime}$ for $\mathrm{C}$ configuration. The ultimate factor limiting the field of view is the diffraction-limited response of the individual antennas.

\footnotetext{
1 http://www.aoc.nrao.edu/ gtaylor/csource.html
}

An approximate formula for the full width at half power (FWHM) of the primary beam (in arcminutes) is: $F W H M=45^{\prime} / v_{\mathrm{GHz}}$. For the highest frequency $(4 \mathrm{GHz})$ in $S$ band, FWHM is approximately $10^{\prime}$.

As NGC 628 is extended over 10.5' in angular size (Table 1), observations with several pointings were needed.

For this purpose, a hexagonal grid was chosen for both $\mathrm{D}$ and $\mathrm{C}$ configurations consisting of 7 pointings, separated by $5^{\prime}$. While the pointing grid is oversampled, this is a safe approach as every position in the galaxy is at least covered three times. In addition, this makes the observations more sensitive to NGC 628's extended disk.

$S$-band is subject to very strong RFI from a number of satellites in particular those providing satellite radio service. A satellite passing through the initial slew will cause unwanted erroneous attenuator settings. Therefore, in order to prevent this, the observation was started with a quick $L$-band scan on $3 \mathrm{C} 48$ which was in a satellite free zone at this time. After this, the $S$-band was switched on.

3C 48 was observed at the start of the observation so it could be used as the main flux density calibrator to calibrate bandpass and absolute flux densities. The source J0240+1848 was observed at least once per hour for use as a phase and polarisation leakage calibrator. 3C 138 was observed twice during the observation in order to calibrate the polarisation angle. Between these calibrator scans, all 7 pointings of the NGC 628 were performed several times. The D and C configuration observations were a total of 5 and $6 \mathrm{~h}$ long.

\subsection{Calibration of JVLA S-band data}

The data were reduced using the Common Astronomy Software Applications (CASA) package ${ }^{2}$ (McMullin et al. 2007). Both D and $\mathrm{C}$ configurations were calibrated identically but separately.

Data affected by shadowed antennas were flagged. As RFI can be extremely strong in $S$-band and as this RFI can produce sharp edges in the spectrum, this can introduce an oscillation across the frequency channels where no RFI is present. This is called Gibbs' phenomenon and can be brought under control by using Hanning smoothing. Due to extreme RFI in several spectral windows at the two extremities of $S$-band, the bandwidths $2.0-2.6 \mathrm{GHz}$ and $3.6-4 \mathrm{GHz}$ were completely flagged. The resulting central frequency is $3.1 \mathrm{GHz}$.

A table of antenna position corrections was produced and incorporated into our calibration using the task GENCAL. A preliminary bandpass correction was applied to the data in order to maximise the effectiveness of the automatic flagging. Visibilities affected by RFI were flagged via the automatic flagging routine

\footnotetext{
2 http://casa.nrao.edu
} 
Table 3. Imaging parameters of the NGC 628 JVLA data.

\begin{tabular}{lcc}
\hline \hline & Natural weighting & \\
\hline Angular resolution & $18^{\prime \prime}(630 \mathrm{pc})$ & \\
Cell size & $3^{\prime \prime}$ & \\
Min \& max cleaning scales & $3.0^{\prime \prime}$ & $180^{\prime \prime}$ \\
\hline & Robust weighting $(0.0)$ & \\
\hline Angular resolution & $7.5^{\prime \prime}(265 \mathrm{pc})$ & \\
Cell size & $1.5^{\prime \prime}$ & \\
Min \& max cleaning scales & $1.5^{\prime \prime}$ & $180^{\prime \prime}$ \\
\hline
\end{tabular}

RFLAG. The antenna EA21 was flagged due to higher than average amplitudes.

The model of 3C 48 was taken from Perley \& Butler (2013a) and was used as our main flux density and bandpass calibrator. The residual delays of each antenna relative to the reference antenna were found using $3 \mathrm{C} 48$. The delays for all antennas were seen to be on average approximately $4 \mathrm{~ns}$ and none were greater than $8 \mathrm{~ns}$. Next, the corrections for the complex antenna gains were obtained. The gain amplitudes were determined by referencing our standard flux density calibrator. To determine the appropriate complex gains for the target source, we used our phase calibrator, J0240+1848. This was the closest and most appropriate phase calibrator that could be found to our target in order to minimise the differences through the ionosphere and troposphere between the phase calibrator and the target source.

For polarisation calibration, the cross-hand delays due to the residual difference between the $\mathrm{R}$ and $\mathrm{L}$ correlations on the reference antenna were solved using 3C 138 which has a higher polarisation signal than $3 \mathrm{C} 48$. Prior to this, the $Q$ and $U$ values were assigned to 3C 138 using the model of Perley \& Butler (2013b).

The phase calibrator $(\mathrm{J} 0240+1848)$ had sufficient parallactic coverage for both observations and was used to solve for polarisation leakage (usually $5 \%$ ).

3C 138 was then used to solve for the polarisation angle. Once this was done, all calibration tables were applied to the calibrators and each pointing for the target. The $\mathrm{C}$ and $\mathrm{D}$ configurations were combined in order to increase the signal-to-noise ratio and maximise the combined uv coverage of the observation.

Self-calibration was tested on the target, however the resulting gain solutions were poor. Applying these solutions degraded the image which was most likely caused by the low signal-tonoise ratio and diffuse nature of this particular target. Therefore, self-calibration was not performed.

\subsection{Imaging and mosaicing}

The Clark CLEAN algorithm (Clark 1980) was used to deconvolve the dirty beam (point spread function; PSF) from the dirty map.

The best strategy found for cleaning the naturally weighted images was to perform CLEAN using a mask until all residuals are cleaned, followed by 20000 clean iterations without a mask and without multi-scale CLEAN. In order to model the extended emission in the field of view, multi-scale CLEAN (Cornwell 2008) was performed with scales ranging from $1.5^{\prime \prime}$ to $180^{\prime \prime}$. A summary of the imaging parameters is given in Table 3. Multi-scale CLEAN is an extension to CLEAN that models the sky brightness by the summation of components of emission having different size scales. The noise for each pointing was found on average to be $5.1 \mu \mathrm{Jy} /$ beam for natural weighting and
$6.9 \mu \mathrm{Jy} /$ beam for robust weighting on average for total intensity (beam sizes can be seen in Table 3). For Stokes $Q$ and $U$, the noise was found to be approximately $4.5 \mu \mathrm{Jy} / \mathrm{beam}$ and $6.5 \mu \mathrm{Jy} /$ beam for natural and robust weighting, respectively.

Multi-frequency synthesis (Rau \& Cornwell 2011) was attempted with the Taylor terms set to 2 . However, it was found that the very weak diffuse emission to the north of the galaxy would be degraded. Leaving the Taylor terms set to 1 was found to produce far better results for this weak extended emission. Unfortunately this means that we are not able to estimate the in-band spectral index, Condon (2015) states that the in-band spectral index for JVLA $S$-band is only reliable for very strong compact sources with a signal-to-noise ratio of $\approx 50$; in addition, the apparent spectral indices of extended sources would be too steep as the synthesised beam solid angle decreases by a factor of four across the $S$-band. In our case, NGC 628 is too weak, diffuse and extended for the in-band spectral index to be reliable.

Each of the seven pointings was imaged separately for each Stokes parameter and then mosaiced together. The primary beam of the final image was constructed from each pointing and applied to the final image. The final image of NGC 628 in total intensity at 7.5" resolution is shown in Fig. 4.

\section{Results from Effelsberg data}

\subsection{Effelsberg maps}

The final images of NGC 628 observed with the Effelsberg telescope are shown in Figs. 1 and 2. The rms noise for total intensity at $8.35 \mathrm{GHz}$ and $2.6 \mathrm{GHz}$ were found to be $0.28 \mathrm{mJy} / \mathrm{beam}$ and $1.1 \mathrm{mJy} /$ beam with their resolutions being $81^{\prime \prime}$ and $4.6^{\prime}$. The average rms noise for Stokes $Q$ and $U$ for $8.35 \mathrm{GHz}$ and $2.6 \mathrm{GHz}$ were found to be $0.10 \mathrm{mJy} / \mathrm{beam}$ and $0.30 \mathrm{mJy} / \mathrm{beam}$, respectively.

At $8.35 \mathrm{GHz}$, NGC 628 is found to be very asymmetric. The most intense emission is seen to the north of the galaxy, at $\mathrm{RA}(\mathrm{J} 2000)=01^{\mathrm{h}} 36^{\mathrm{m}} 41^{\mathrm{s}}, \operatorname{Dec}(\mathrm{J} 2000)=+15^{\circ} 48^{\prime} 29^{\prime \prime}$ rather than the centre of the galaxy, suggesting that NGC 628 does not have an active nucleus like M 51 (Rampadarath et al. 2015). The brightest region of NGC 628 is part of the northern spiral arm, consisting of many $\mathrm{H}_{\mathrm{II}}$ regions, and is also bright in the infrared spectral range (Kennicutt et al. 2011) and in $\mathrm{H}_{\mathrm{I}}$ line emission (Walter et al. 2008). This agrees with the observation of Marcum et al. (2001) that the inner regions of the galaxy have experienced more rapidly declining star formation than the outer regions.

In linear polarisation, the $8.35 \mathrm{GHz}$ map shows diffuse emission along with the polarisation angles forming a spiral pattern. A polarised background source $(\mathrm{J} 013657+154422$, shown with the letter A in Fig. 1) is apparent at the south-east of the galaxy. There is no sign of polarisation in the central region of the galaxy, probably due to beam depolarisation.

On the $2.6 \mathrm{GHz}$ map, no significant features can be made out in total intensity, owing to the poor resolution of the Effelsberg telescope at this frequency. The total intensity emission is seen to extend to the southwest due to an unresolved background source (seen as the letter $C$ in Figs. 2 and 4). Significant polarisation is detected, with the polarisation angles creating a spiral pattern. No polarisation can be seen in the central region. The strongest polarised emission is seen again at the location of J013657+154422 (letter A in Fig. 2 (right)) but significant diffuse polarised emission is also observed. Most likely this emission originates from the inter-arm regions of NGC 628 which will be become apparent when inspecting the JVLA data. 

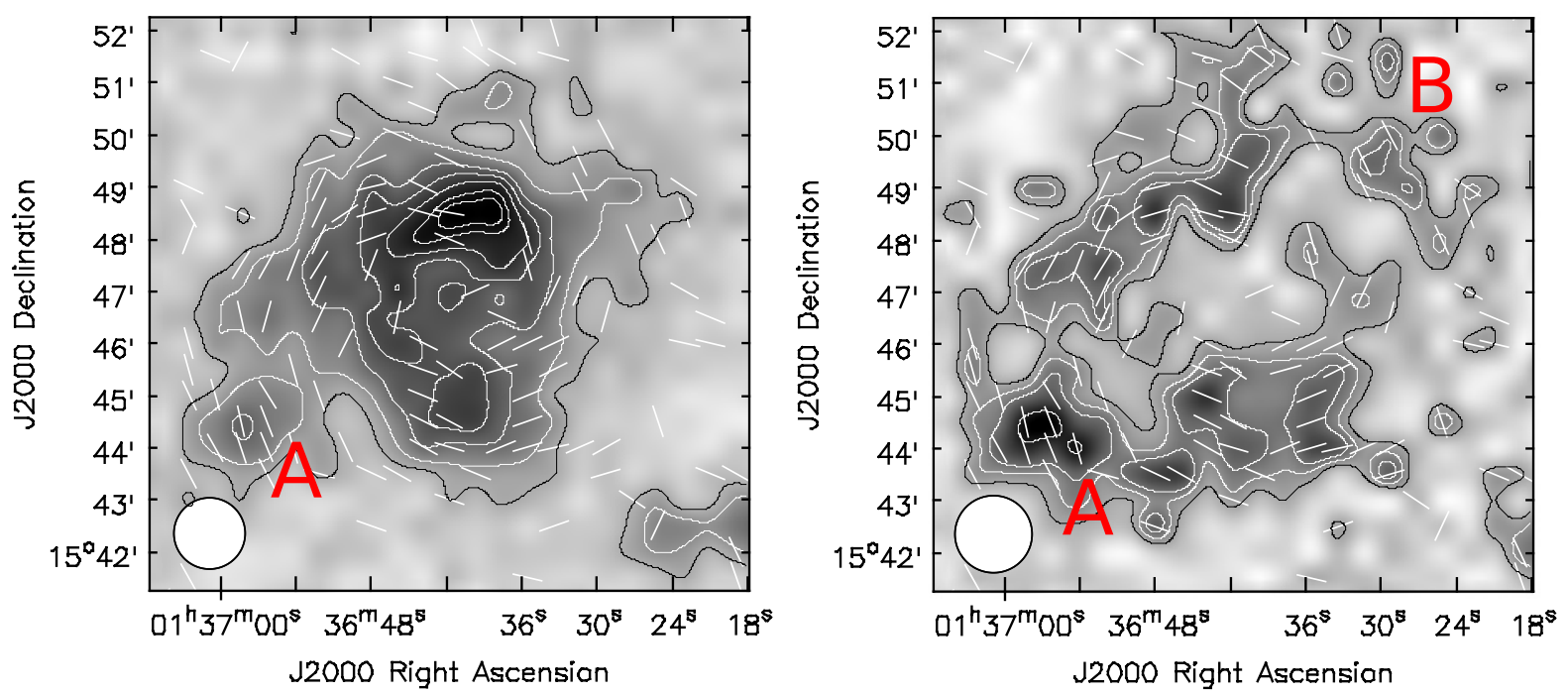

Fig. 1. NGC 628 observed at $8.35 \mathrm{GHz}$ with the Effelsberg $100-\mathrm{m}$ telescope at a resolution of $81^{\prime \prime}$. Left image: total intensity of NGC 628 with contours representing $3,5,8,10,12,14,15 \times 30 \mu \mathrm{Jy} /$ beam. Right image: linearly polarised intensity of NGC 628 with contours representing 3 , 4 , $5,8 \times 60 \mu \mathrm{Jy} /$ beam. The vectors show $E+90^{\circ}$, not corrected for Faraday rotation, which is very small at this frequency. All vectors are plotted with the same length.
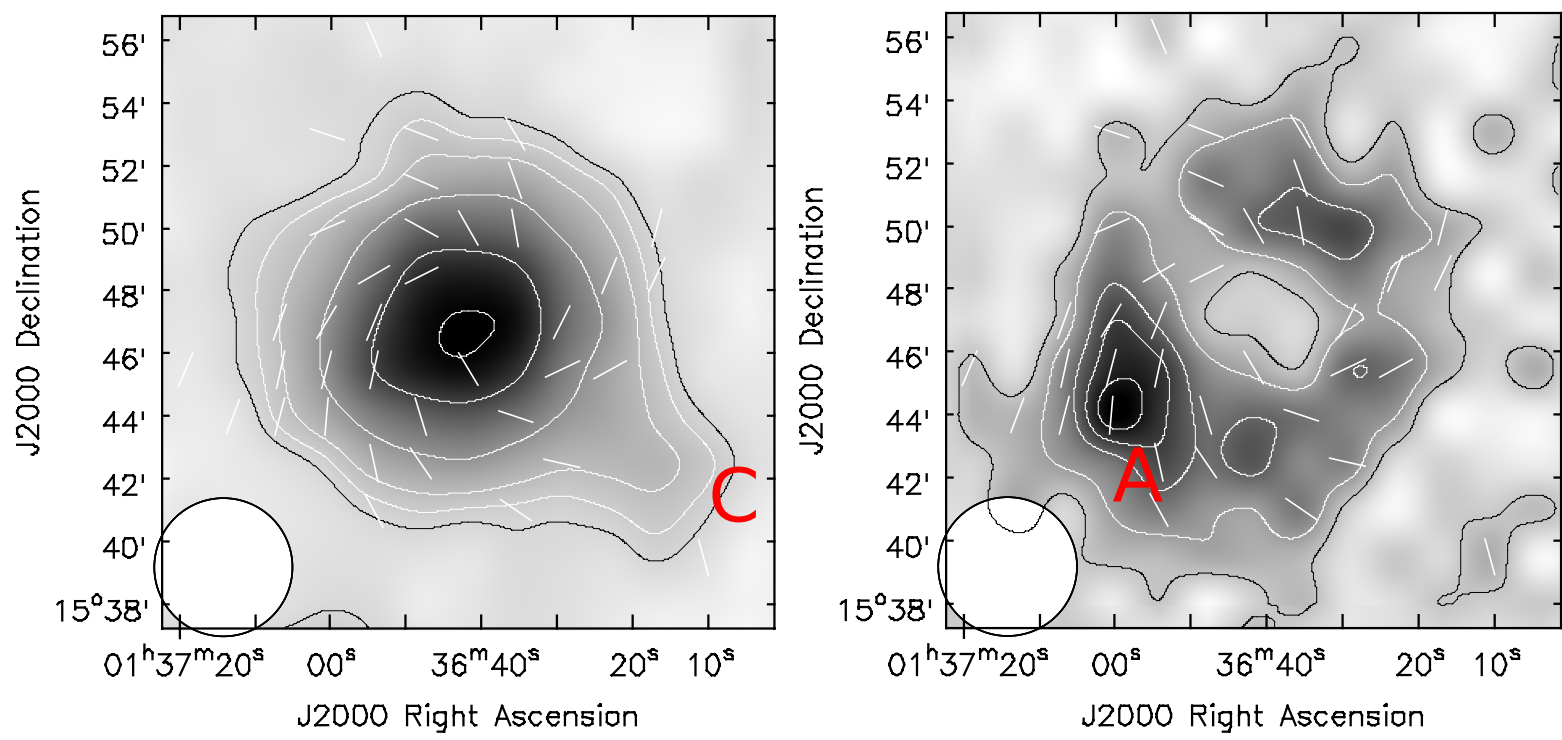

Fig. 2. NGC 628 observed at $2.65 \mathrm{GHz}$ with the Effelsberg $100-\mathrm{m}$ telescope at a resolution of $4.6^{\prime}$. Left image: total intensity of NGC 628 with contours representing $3,5,8,16,32,44 \times 1 \mathrm{mJy} /$ beam. Right image: linearly polarised intensity of NGC 628 with contours representing $3,5,8$, $10,12 \times 2.5 \mathrm{mJy} /$ beam. The vectors show $E+90^{\circ}$, not corrected for Faraday rotation, which is small at this frequency. All vectors are plotted with the same length.

\subsection{Integrated radio continuum spectral analysis}

The integrated flux densities found for NGC 628 are $38 \pm 3 \mathrm{mJy}$ at $8.35 \mathrm{GHz}$ and $110 \pm 10 \mathrm{mJy}$ at $2.6 \mathrm{GHz}$. Measurements from the literature (Table 4) were also used to determine the spectral index of the galaxy. All these measurements are tied to the flux scale of Baars et al. (1977). A single power-law fit to the data yields a spectral index of $\alpha=-0.79 \pm 0.06$. A plot of this data can be seen in Fig. 3. This value not only agrees with Paladino et al. (2009; $\alpha=-0.78)$ but is a normal value for spiral galaxies ( $\alpha=$ $-0.74 \pm 0.03$, Gioia et al. 1982).

At low frequencies, the spectrum of NGC 628 shows no indications of flattening all the way down to $57 \mathrm{MHz}$. This suggests that neither free-free absorption of the synchrotron emission nor ionisation losses are significant for the galaxy as a whole. The galaxy's integrated spectrum remains a power law, probably due to the clumpy nature of the interstellar medium (ISM: Basu et al. 2015).

A straight power-law spectrum was also seen for M 51 down to $151 \mathrm{MHz}$ (Mulcahy et al. 2014). It should be noted that the flux density of NGC 628 from Israel \& Mahoney (1990) is uncertain due to the poor sensitivity and angular resolution of the Clark Lake telescope and should be treated cautiously. Without this integrated flux, the fitted spectral index is found to be $\alpha=-0.79 \pm 0.03$. Unfortunately, no other integrated fluxes has been taken at these low frequencies. Observations with LOFAR (van Haarlem et al. 2013) below $100 \mathrm{MHz}$ are underway and will help to verify whether the integrated spectrum is indeed a straight power law but will additionally be able to resolve this galaxy to arcsecond resolution. 
Table 4. Integrated flux densities of NGC 628.

\begin{tabular}{ccc}
\hline \hline$v(\mathrm{GHz})$ & Flux density (Jy) & Reference \\
\hline 8.35 & $0.038 \pm 0.003$ & This work \\
4.85 & $0.06 \pm 0.005$ & Paladino et al. (2009) \\
2.614 & $0.11 \pm 0.01$ & This work \\
1.515 & $0.16 \pm 0.01$ & Paladino et al. (2009) \\
1.4 & $0.15 \pm 0.01$ & Condon et al. (1998) \\
0.324 & $0.49 \pm 0.03$ & Paladino et al. (2009) \\
0.057 & $2 \pm 1$ & Israel \& Mahoney (1990) \\
\hline
\end{tabular}

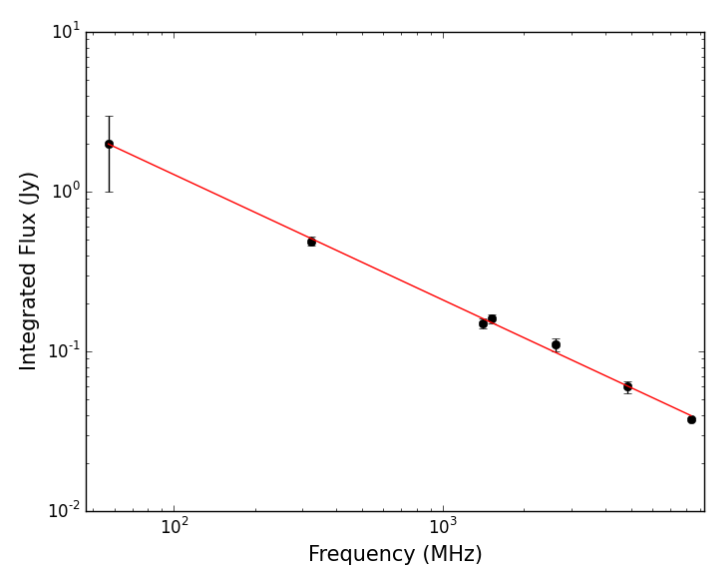

Fig. 3. Integrated spectrum of NGC 628 using Effelsberg and literature integrated flux measurements with the red line representing a single power law with a spectral index of $\alpha=-0.79 \pm 0.06$.

\section{Results from JVLA S-band data}

\subsection{Total intensity}

The final total intensity image produced with robust weighting at $7.5^{\prime \prime}$ resolution is shown in Fig. 4, with robust weighting and smoothed to $10^{\prime \prime}$ resolution in Fig. 5 (upper image), and with natural weighting at $18^{\prime \prime}$ resolution in Fig. 6 (upper image).

The integrated flux density of the JVLA data at $3.1 \mathrm{GHz}$ is approximately $120 \mathrm{mJy}$. This is comparable to the Effelsberg observation at $2.6 \mathrm{GHz}$ (Table 4), demonstrating that the flux density calibration was successful and that there is no significant flux density loss due to the missing small spacings of the JVLA.

Weak radio emission surrounds NGC 628 and should be mostly nonthermal in nature (Fig. 6, upper panel). Two main radio spiral arms are present, both coiling out in a counterclockwise fashion. One spiral arm dominates the north and east of the galaxy ("outer arm") with the most intense emission located at $\operatorname{RA}(\mathrm{J} 2000)=01^{\mathrm{h}} 36^{\mathrm{m}} 38^{\mathrm{s}}, \operatorname{Dec}(\mathrm{J} 2000)=+15^{\circ} 48^{\prime} 47^{\prime \prime}$, corresponding to a cluster of $\mathrm{H}$ in regions which are unresolved in the radio image. This region shows strong emission in $\mathrm{H} \alpha$ (Kennicutt et al. 2003) and UV (Marcum et al. 2001), in addition to $\mathrm{H}_{\mathrm{I}}$ (Walter et al. 2008). The outer arm branches out into two components in the east (Fig. 5, upper image).

The second main spiral arm ("inner arm") travels counterclockwise from north to the south; several $\mathrm{H}$ II complexes are located along the radio spiral arm (Fig. 6, upper panel). These so-called "beads on a string" are bright knots embedded in diffuse emission tracing the spiral pattern. Many of the UV-bright knots are also bright in $\mathrm{H} \alpha$ indicating recent (less than $100 \mathrm{Myr}$ ) star formation and the radio continuum matches very well to the brightest of these knots. These are especially evident in Fig. 4 where the resolution is high enough to resolve these individual knots. The brightest of these knots are located on the inside edges of the spiral arms formed by the diffuse UV continuum. The youngest and most massive stars are located at the inner edge of the pattern (Chen et al. 1992; Cornett et al. 1994; Marcum et al. 2001) which are the knots observed at $3.1 \mathrm{GHz}$. The inner arm continues to the west where the flux density of the arm suddenly decreases and becomes indistinguishable from the radio envelope of the galaxy, with exception of the $\mathrm{H}_{\text {II }}$ regions located at RA $(J 2000)=01^{\mathrm{h}} 36^{\mathrm{m}} 29^{\mathrm{s}}, \operatorname{Dec}(\mathrm{J} 2000)=+15^{\circ} 48^{\prime} 50^{\prime \prime}$.

To the west of the galaxy, three narrow radio filaments are seen (marked $\mathrm{Wa}, \mathrm{Wb}$ and $\mathrm{Wc}$ in Fig. 5, upper image), all with a width of approximately $900 \mathrm{pc}$. The most southern filament is an extension of the inner arm. No major $\mathrm{H}$ il regions exist in these regions and therefore the emission is most likely nonthermal.

Unlike most nearby galaxies observed in radio continuum, for example M 51 (Mulcahy et al. 2014), IC 342 (Beck 2015) and NGC 6946 (Beck 2007), NGC 628 shows no trace of a central source, while this is similar to the flocculent galaxy NGC 4414 (Soida et al. 2002).

\subsection{Comparison to $\mathrm{H}$ I observations}

Throughout the disk, there are regions where little to no radio emission is seen, most notably the holes located at RA(J2000) = $01^{\mathrm{h}} 36^{\mathrm{m}} 48^{\mathrm{s}}, \operatorname{Dec}(\mathrm{J} 2000)=+15^{\circ} 45^{\prime} 10^{\prime \prime}$ and $\mathrm{RA}(\mathrm{J} 2000)=$ $01^{\mathrm{h}} 36^{\mathrm{m}} 50^{\mathrm{s}}, \operatorname{Dec}(\mathrm{J} 2000)=+15^{\circ} 46^{\prime} 27^{\prime \prime}$ (shown in Figs. 6 and 7 as Roman numerials). These holes in radio emission correspond to $\mathrm{H}_{\mathrm{I}}$ holes detected by Bagetakos et al. (2011; Fig. 7). Several more holes can be seen in the west of the galaxy. A list of $\mathrm{HI}_{\mathrm{I}}$ holes corresponding to holes in radio continuum and their physical parameters (from Bagetakos et al. 2011) is given in Table 5. All the holes seen are of type 1 which is a hole where the gas has been completely blown out of the disk of the galaxy. Approximately $75 \%$ of all $\mathrm{H}_{\mathrm{I}}$ holes are type 1 .

Such holes in total radio continuum corresponding to an $\mathrm{H}$ I hole have been seen previously in NGC 6946 by Beck (2007) who mentioned that the association between an $\mathrm{H}$ i hole and radio continuum is rare and that the lack of radio emission could be due to two main reasons. The first is that the superbubble creating the H I holes, which are driven by multiple supernova explosions, are sweeping away the gas and magnetic field and thereby creating a locally weak magnetic field. The second explanation is that the superbubble carries the magnetic field vertically into the halo along with the hot gas (Norman \& Ikeuchi 1988). As NGC 628 is nearly exactly face-on compared to NGC 6946 ( $i=$ $33^{\circ}$ ), these vertical fields would be along the line of sight, therefore producing no observable synchrotron emission but possibly strong Faraday rotation (see Sect. 5.6).

The larger number of $\mathrm{H}_{\mathrm{I}}$ holes corresponding to holes in the radio continuum in NGC 628 compared to NGC 6946 could be an effect of the different inclinations. When viewing a $\mathrm{H}_{\mathrm{I}}$ hole carrying a magnetic field in NGC 6946 vertical to the disk with its inclination of approximately $33^{\circ}$, we observe variations of the magnetic field component perpendicular to the line of sight which would give rise to observable synchrotron emission. This would occur less likely for NGC 628 with its smaller inclination.

The Hi holes seen in NGC 628 all have a kinetic age greater than $40 \mathrm{Myr}$ and should be old enough so that the field configuration has gained a significant vertical offset (Heald 2012). However, two holes are significantly older and the vertical shear should have destroyed the vertical offset of the magnetic field. Notably, these holes are the most obvious in our radio continuum image (Fig. 7). While the vertical shear of NGC 628 is not known 
Table 5. H I holes corresponding to holes in radio continuum and their physical parameters.

\begin{tabular}{cccccc}
\hline \hline $\begin{array}{c}\text { RA } \\
(\mathrm{h} \mathrm{m} \mathrm{s})\end{array}$ & $\begin{array}{c}\text { Dec } \\
\left({ }^{\circ}{ }^{\prime}{ }^{\prime \prime}\right)\end{array}$ & $\begin{array}{c}\text { Diameter } \\
(\mathrm{pc})\end{array}$ & $\begin{array}{c}t_{\mathrm{kin}} \\
(\mathrm{Myr})\end{array}$ & $\begin{array}{c}\log \left(E_{\mathrm{E}}\right) \\
\left(10^{50} \mathrm{erg}\right)\end{array}$ & $\begin{array}{c}\log \left(M_{\mathrm{HI}}\right) \\
\left(10^{4} M_{\odot}\right)\end{array}$ \\
\hline 013628.7 & +154642.0 & 878 & 61 & 3.0 & 2.8 \\
013631.1 & +154826.9 & 814 & 57 & 2.9 & 2.7 \\
013631.4 & +154720.9 & 585 & 41 & 2.4 & 2.3 \\
013632.8 & +154746.4 & 901 & 63 & 3.0 & 2.6 \\
013633.2 & +154656.8 & 743 & 52 & 2.8 & 2.5 \\
013635.0 & +154720.8 & 888 & 62 & 3.1 & 2.6 \\
013635.4 & +154925.3 & 758 & 53 & 2.7 & 2.6 \\
013647.8 & +154511.4 & 1782 & 125 & 4.1 & 3.4 \\
013650.5 & +154630.8 & 1573 & 110 & 4.0 & 3.3 \\
\hline
\end{tabular}

Notes. $t_{\text {kin }}, E_{\mathrm{E}} \& M_{\mathrm{HI}}$ are the kinetic age, energy requirement, and missing $\mathrm{H}_{\mathrm{I}}$ mass of the $\mathrm{H}_{\mathrm{I}}$ hole (from Bagetakos et al. 2011).

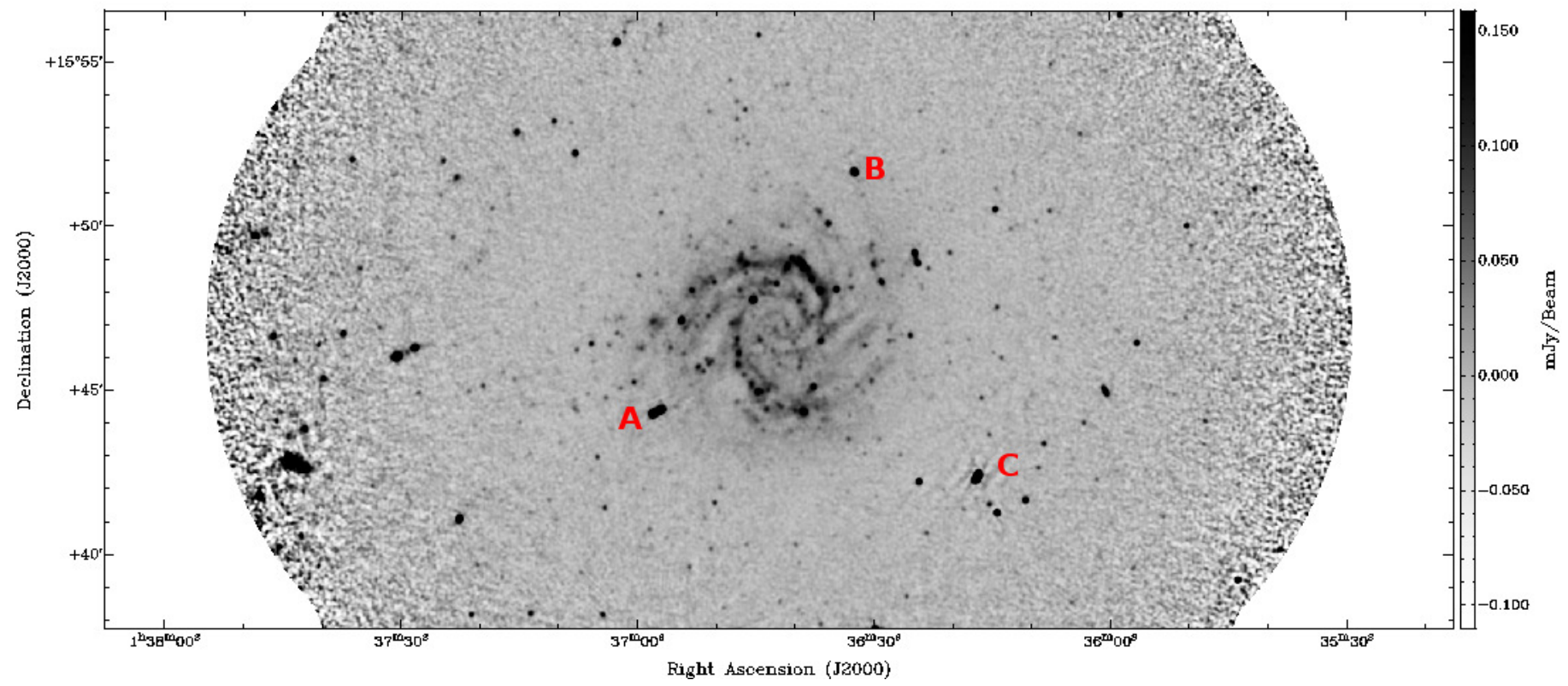

Fig. 4. Total intensity map of NGC 628 at $3.1 \mathrm{GHz}$ at $7.5^{\prime \prime}$ resolution, observed with the combined D \& C configurations of the JVLA. The rms noise is approximately $5 \mu \mathrm{Jy} /$ beam. This image has been primary beam corrected. Several background sources are labelled for future reference.

to the authors' knowledge, Heald et al. (2007) found that the vertical shear for a sample of three galaxies is approximately 15$25 \mathrm{~km} \mathrm{~s}^{-1}$ per scale height in $\mathrm{H} \alpha$, independent of radius. This gives a lower limit of $60 \mathrm{Myr}$ for a characteristic shear time. Therefore the first argument is more likely to be true, especially for the two H I holes in question shown in Fig. 7, namely that the gas and magnetic field have been radially swept away, leaving a weak local magnetic field.

To the north of the galaxy, extended, low-level emission is observed (Fig. 6, top), reaching out to $13 \mathrm{kpc}$ from the centre of the galaxy. The extension is seen to the north-north-west direction. Significant $\mathrm{H}_{\mathrm{I}}$ emission is present in this region and the radio emission overlays well with the extended northern $\mathrm{H}$ i arm (Fig. 8). Additionally, there are plenty of $\mathrm{H}$ II regions located in the extended disk of NGC 628. Many of these H II regions are located on the Hi arm (Ferguson et al. 1998; Lelièvre \& Roy 2000). H II regions in the inner disk usually have a (SFR) of $\approx 10^{3}$ higher than that of the faintest $\mathrm{H}_{\text {II }}$ regions in the outer disk. Therefore, it is most likely that we are observing cosmic ray electrons (CREs) originating from supernovae occurring at these faint $\mathrm{H}$ iI knots from this extreme northern spiral arm.

\subsection{Separation of thermal emission}

For an accurate determination of the magnetic field strength, separating the two components of continuum emission, namely free-free (thermal) and synchrotron (nonthermal) needs to be performed. There are several methods, the classical approach where a constant nonthermal spectral index is assumed (e.g. Klein et al. 1984) or using the $24 \mu \mathrm{m}$ infrared emission to directly calculate the thermal emission (Murphy et al. 2008). For this work we shall apply the method by Tabatabaei et al. (2007a) where an extinction-corrected $\mathrm{H} \alpha$ map is used to estimate the thermal emission. The continuum-subtracted $\mathrm{H} \alpha$ map used was obtained from the ancillary data at the SINGS (Kennicutt et al. 2003) website. The maps were in units of $\mathrm{DN} \mathrm{s}^{-1}$ pixel $^{-1}$ which was converted into erg s $\mathrm{s}^{-1} \mathrm{~cm}^{-2}$ using the calibration provided in the SINGS fifth data delivery documentation ${ }^{3}$.

First of all, the dust temperature was calculated using 70 and $160 \mu \mathrm{m}$ Herschel maps (Kennicutt et al. 2011). All maps were smoothed to a resolution of $18^{\prime \prime}$ and normalised to a common grid. Both IR maps were calibrated in surface brightness units

\footnotetext{
3 https://irsa.ipac.caltech.edu/data/SPITZER/SINGS/ doc/sings_fifth_delivery_v2.pdf
} 

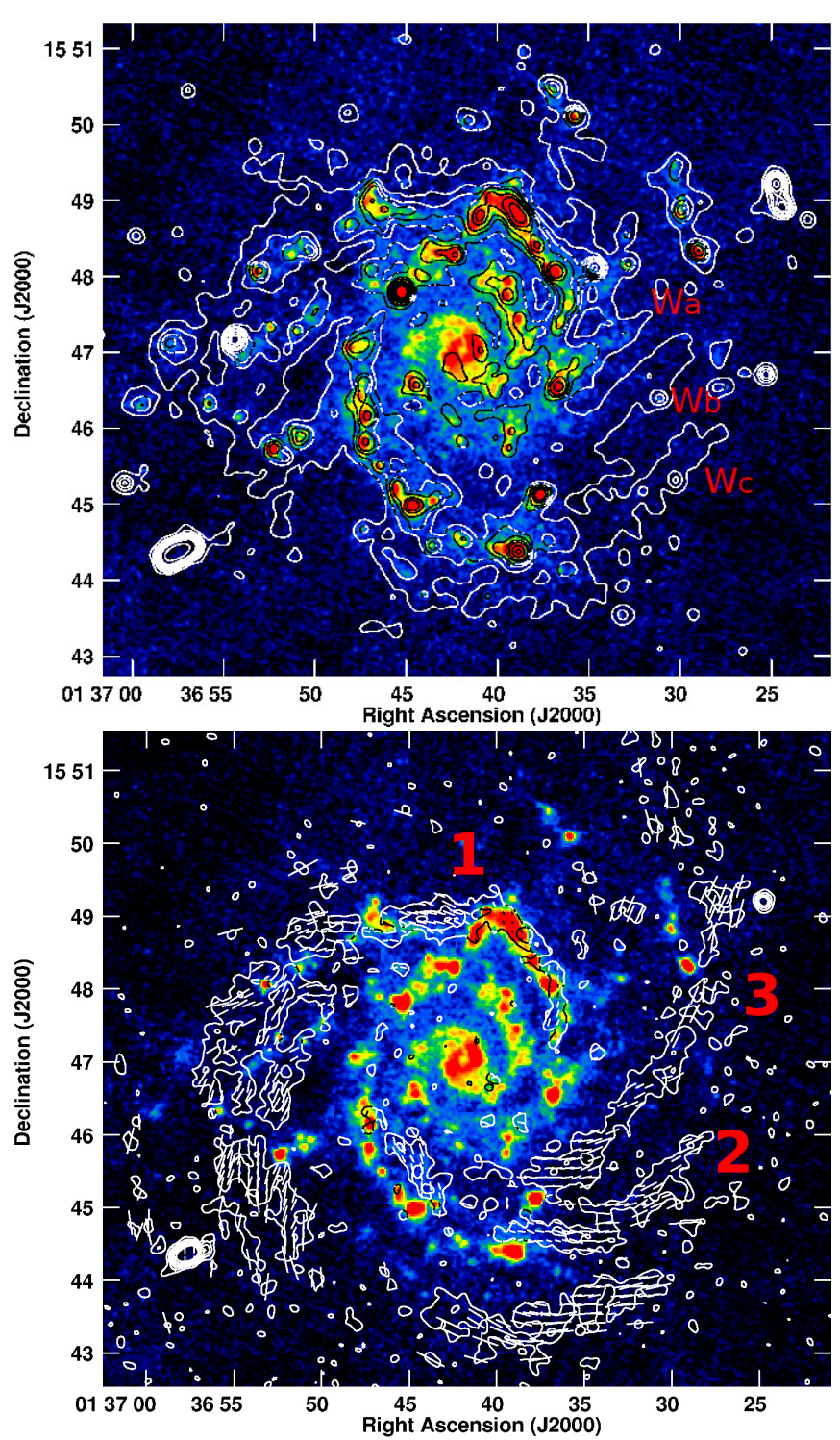

Fig. 5. Robust-weighted JVLA image of NGC 628 of total intensity (top) and linearly polarised intensity (bottom) at $3.1 \mathrm{GHz}$ at a resolution of $10^{\prime \prime}$, averaged over all channels, and overlaid onto an $70 \mu \mathrm{m} \mathrm{IR}$ image from Herschel (Kennicutt et al. 2011). Contours of total intensity are at $1,2,3,4,6,8,12,16,32,64,128 \times 40 \mu \mathrm{Jy} /$ beam. The nonthermal arms to the west are marked Wa to Wc for future reference. Contours of polarised intensity are at $1,2,3,4,6,8,12 \times 12 \mu \mathrm{Jy} / \mathrm{beam}$. The white lines show the magnetic field orientations $\left(E+90^{\circ}\right)$, not corrected for Faraday rotation, with a length of $10^{\prime \prime}$ representing $30 \%$ degree of polarisation. The main polarised arms are marked from 1 to 3 for future reference.

of $\mathrm{MJy} \mathrm{sr}^{-1}$. The mean dust temperature across the galaxy was found to be $22.4 \mathrm{~K}$, very similar to NGC 6946 with a mean dust temperature of $22.3 \mathrm{~K}$ (Basu et al. 2012). A histogram showing the distribution of dust temperatures is shown in Fig. 9.

In the brightest $\mathrm{H}$ II regions, the dust temperature is $25 \mathrm{~K}$, in the central region and areas of the spiral arms $23 \mathrm{~K}$, and in other regions approximately $21 \mathrm{~K}$. From $T_{\text {dust }}$, the optical depth at $160 \mu \mathrm{m}$ was derived from Eq. (2) in Tabatabaei et al. (2007a). The $\mathrm{H} \alpha$ optical depth was calculated using the equation $\tau_{\mathrm{H} \alpha} \approx$ $f_{\mathrm{d}} \times 2200 \times \tau_{160 \mu \mathrm{m}}$ (Krügel 2003) assuming an $\mathrm{H} \alpha$ filling factor of 0.33 (Dickinson et al. 2003). This optical depth was then used to de-redden the $\mathrm{H} \alpha$ flux density, using Eq. (3) in Tabatabaei et al. (2007a).
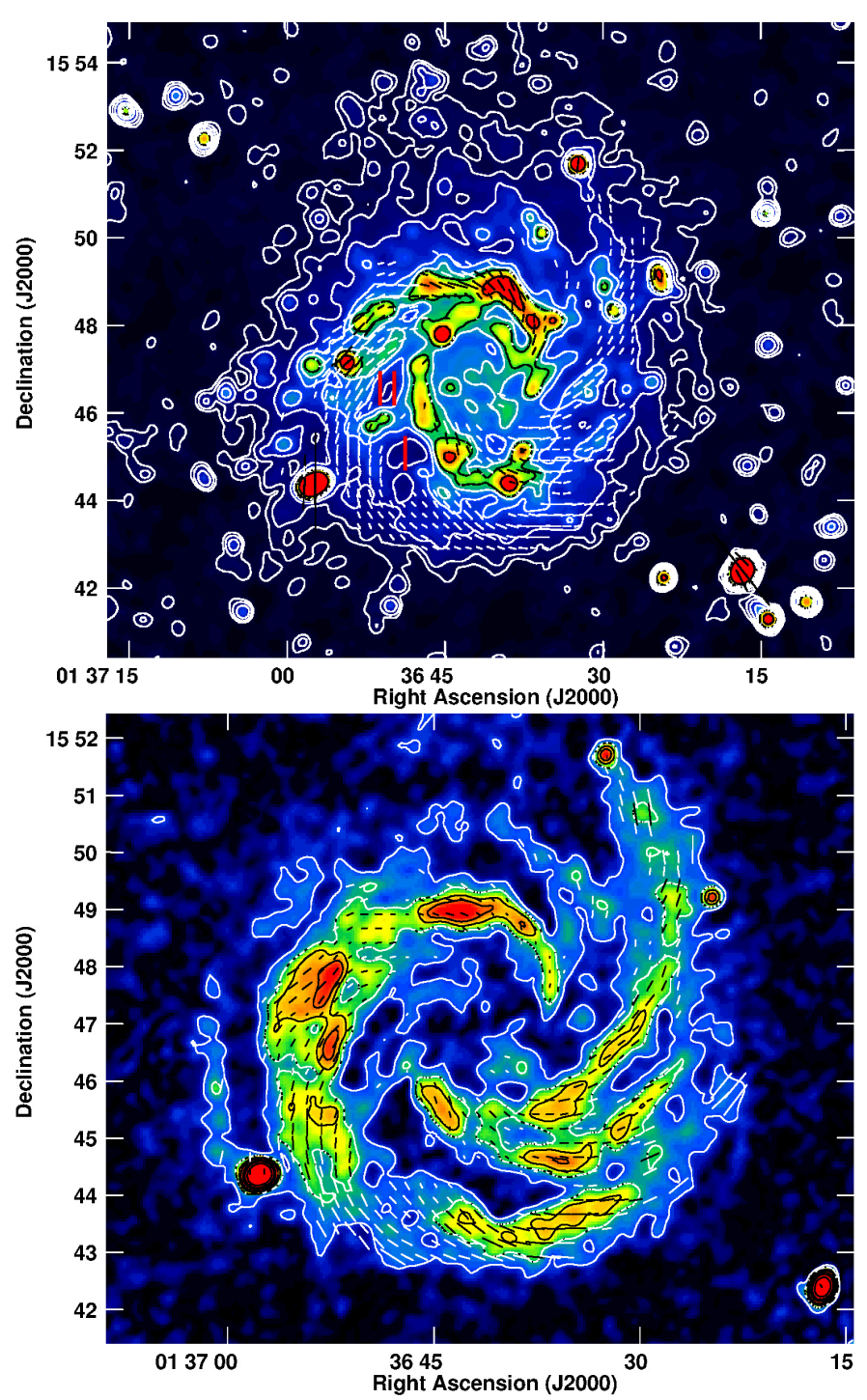

Fig. 6. Natural-weighted JVLA image of NGC 628 of total intensity (top) and linearly polarised intensity (bottom) at $3.1 \mathrm{GHz}$ at a resolution of $18^{\prime \prime}$, averaged over all channels. Contours of total intensity are at 1 , $2,4,8,12,16,32 \times 20 \mu \mathrm{Jy} /$ beam. Contours of polarised intensity are at $1,2,3,4,8,12 \times 15 \mu \mathrm{Jy} /$ beam. The lines show the magnetic field vectors $\left(E+90^{\circ}\right)$, not corrected for Faraday rotation, with a length of $10^{\prime \prime}$ representing a polarised intensity of $150 \mu \mathrm{Jy} /$ beam (top) and 30\% degree of polarisation (bottom), respectively. The roman numerals I and II refer to the $\mathrm{H}_{\mathrm{I}}$ holes shown in Fig. 7.

From the de-redded $\mathrm{H} \alpha$ map, the emission measure (EM) was found using Eq. (4) in Tabatabaei et al. (2007a), assuming an electron temperature of $10^{4} \mathrm{~K}$ (Valls-Gabaud 1998). Equations (5) and (6) from Tabatabaei et al. (2007a) were used to calculate the continuum optical depth and brightness temperature $T_{\mathrm{B}}$. The thermal flux density was then obtained from $T_{\mathrm{B}}$, using the equation in Basu et al. (2012):

$\frac{S_{v, \text { th }}}{\mathrm{Jy} \mathrm{beam}^{-1}}=8.18 \times 10^{-7}\left(\frac{\theta_{\text {maj }}}{\operatorname{arcsec}}\right)\left(\frac{\theta_{\text {min }}}{\operatorname{arcsec}}\right)\left(\frac{v}{\mathrm{GHz}}\right)\left(\frac{T_{\mathrm{B}}}{\mathrm{K}}\right)$,

where $\theta_{\text {maj }}$ and $\theta_{\min }$ are the major and minor axis of the synthesised beam, respectively.

Finally, the nonthermal map at $3.1 \mathrm{GHz}$ is obtained by subtracting the thermal emission map from the JVLA 3.1 GHz naturally weighted map (Fig. 6, top). When calculating the nonthermal map for regions where there is no observable dust emission, 


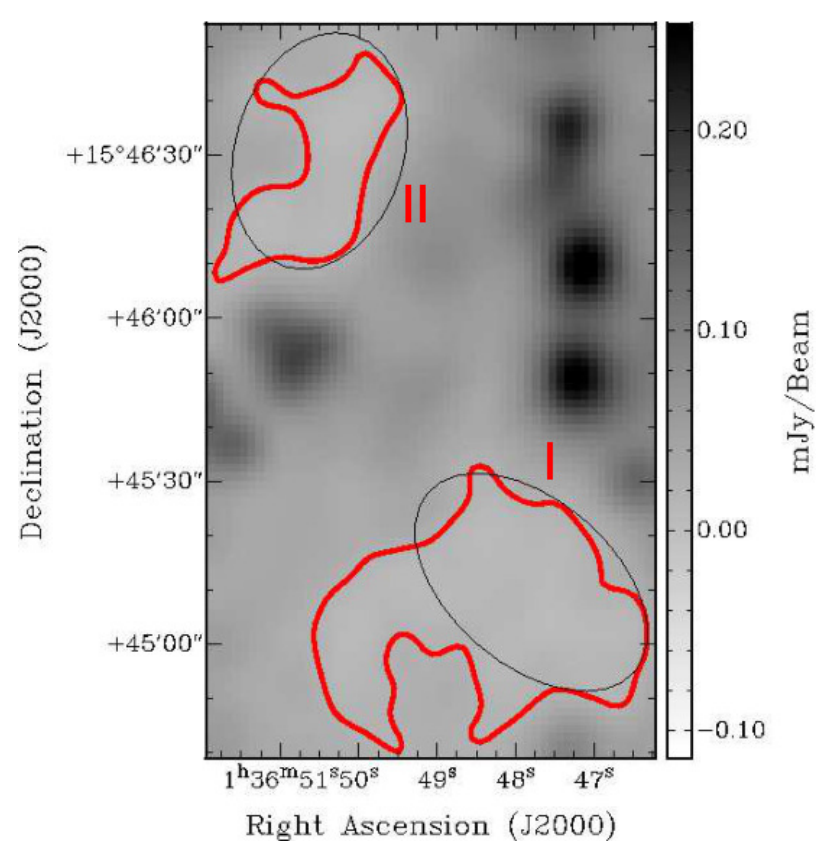

Fig. 7. Overlay of two prominent $\mathrm{H}_{\mathrm{I}}$ holes on the radio continuum image (Fig. 5) at 10" resolution. The contour level is at $21 \mu \mathrm{Jy} / \mathrm{beam}$ which is approximately $3 \sigma$.

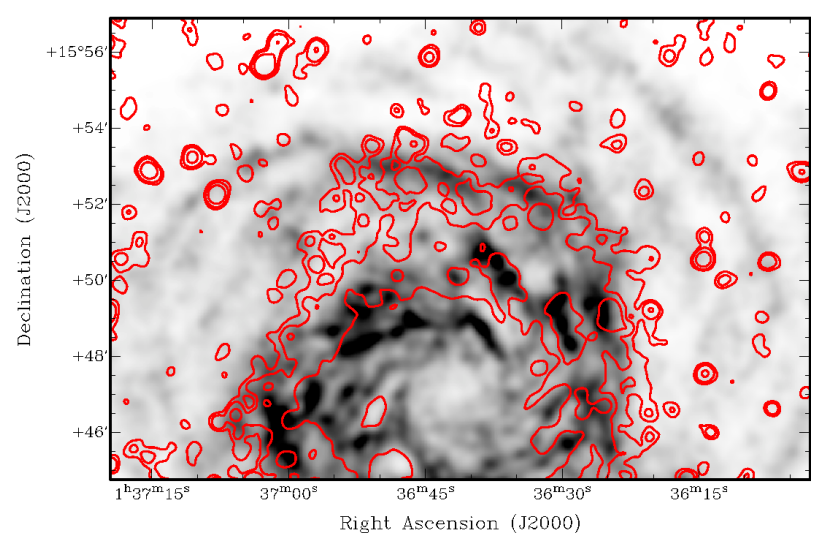

Fig. 8. Overlay of the radio continuum emission (red contours) to the

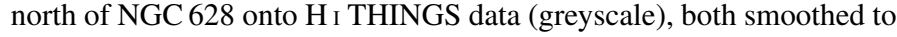
the same resolution of $18^{\prime \prime}$. Contours are at $20,40,120 \mu \mathrm{Jy} / \mathrm{beam}$.

we set the thermal fraction to zero. This assumption is not entirely true as there could be undetected dust IR emission present in the Hershel map. Both the thermal and nonthermal maps of NGC 628 at $3.1 \mathrm{GHz}$ are shown in Fig. 10.

The map of the thermal fraction created from the nonthermal and thermal maps of NGC 628 is shown in Fig. 11. The spiral arms show a thermal fraction of $10-20 \%$, with typical H II regions showing $20-30 \%$ and the largest $\mathrm{H}_{\text {II }}$ regions having thermal fractions greater than $40 \%$.

The very centre of the galaxy is observed to have thermal fractions that reach up to $47 \%$, indicating that a significant fraction of the emission in this region is of thermal origin. This could be explained by a lack of cosmic ray electrons originating from supernovae in this region, comparable to the central region of M31 where Tabatabaei et al. (2013) found the thermal fraction to be approximately $20 \%$ at $\lambda 20 \mathrm{~cm}$ due to weak synchrotron emission caused by a lack of cosmic ray electrons (CREs).

Cornett et al. (1994), utilising both far and near UV photometry images, found that NGC 628's central region shows no

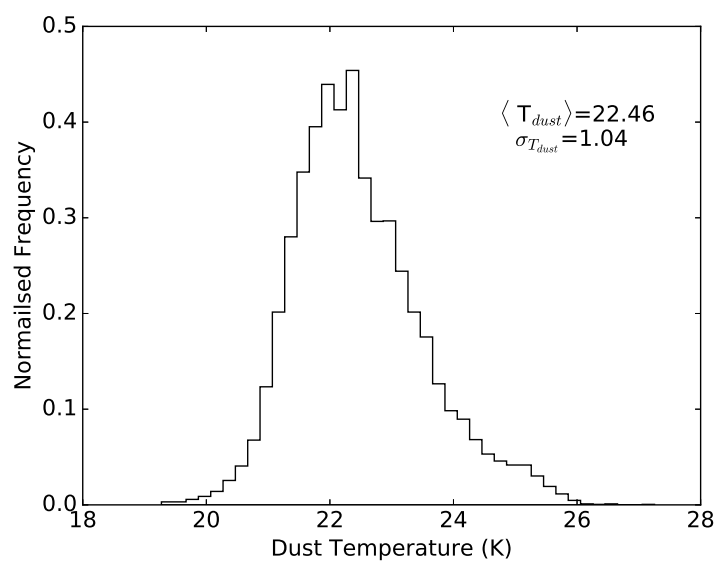

Fig. 9. Histogram displaying the pixel-wise distribution of dust temperatures.

significant population of OB stars. They also observed that the azimuthally averaged scale lengths for the UV continuum emission decreases with increasing wavelength. However, the UV profiles are clearly non-exponential, despite the approximately exponential behaviour of the $R$-band profile. Analysing these colour gradients, Cornett et al. (1994) concluded that they reflect the star formation history rather than metallicity or internal extinction. Together with findings from Marcum et al. (2001), we conclude that the entire disk has undergone active star formation within the past $500 \mathrm{Myr}$ but the inner regions have experienced a more rapidly declining star formation than the outer regions.

The Initial Mass Function (IMF) seems to be universal (Kroupa 2001, 2002), while its form has only been determined directly on star cluster scales. This canonical IMF has traditionally been applied on galaxy-wide scales and has to be constructed by adding all young stars of all young star clusters (Kroupa \& Weidner 2003; Weidner \& Kroupa 2006). This integrated galactic initial mass function (IGIMF) is steeper than the usual IMF in star clusters and steepens with decreasing total SFR (Weidner \& Kroupa 2005; Pflamm-Altenburg et al. 2007). This is due to the combination of two effects. The first is that the most massive star in a star cluster is a function of the total stellar mass of the young embedded star cluster (Weidner \& Kroupa 2005). The second is that the most massive young embedded star cluster is a function of the total SFR of a galaxy (Weidner et al. 2004). Similar to the IMF in star clusters, the embedded cluster mass function (ECMF), which describes the mass spectrum of newly formed star clusters, follows a power-law distribution function in galaxies (Lada \& Lada 2003). Therefore low-mass clusters do not contain massive stars, and this yields an IGIMF that depends on the SFR, since the most massive cluster that can form depends on the SFR.

For NGC 628, we see UV emission from the central region from the B stars which are still there and can ionise the gas and give rise to $\mathrm{H} \alpha$ emission.This is also observed in M31 as high mass stars appear to be ruled out as the primary source for $\mathrm{H} \alpha$ emission in the inner region (Devereux et al. 1994). However, the current IGIMF lacks O stars if the SFR is very low, such that Type II supernova explosions will not be occurring. No radio synchrotron emission is visible because only $\mathrm{O}$ stars produce Type II supernovae which in turn generate CREs emitting radio synchrotron emission.

Star formation continues in the spiral arms where we have both $\mathrm{O}$ and $\mathrm{B}$ stars, both contributing to the UV emission and giving rise to radio synchrotron emission. In the outer regions 

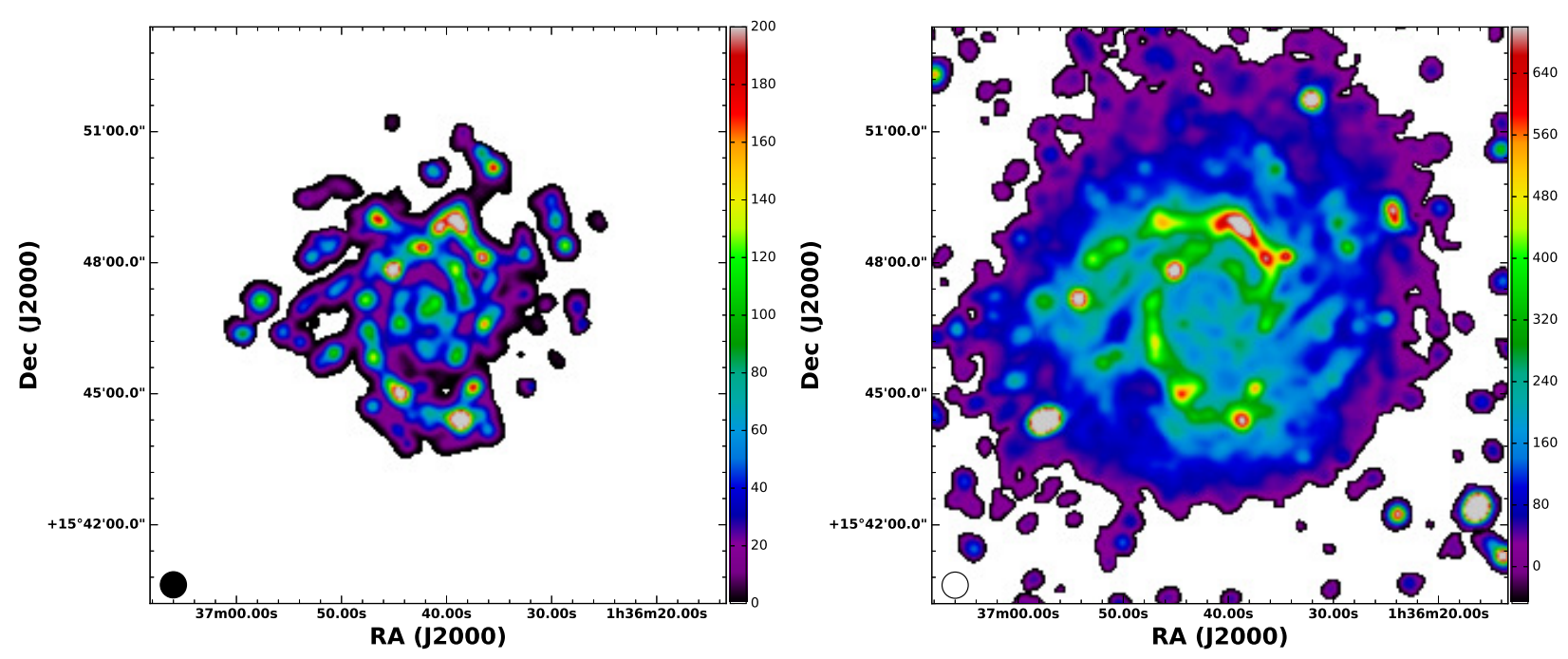

Fig. 10. Images of thermal (left) and nonthermal intensity (right) of NGC 628 at $3.1 \mathrm{GHz}$ at $18^{\prime \prime}$ resolution. Colour scale is in $\mu \mathrm{Jy} / \mathrm{beam}$.

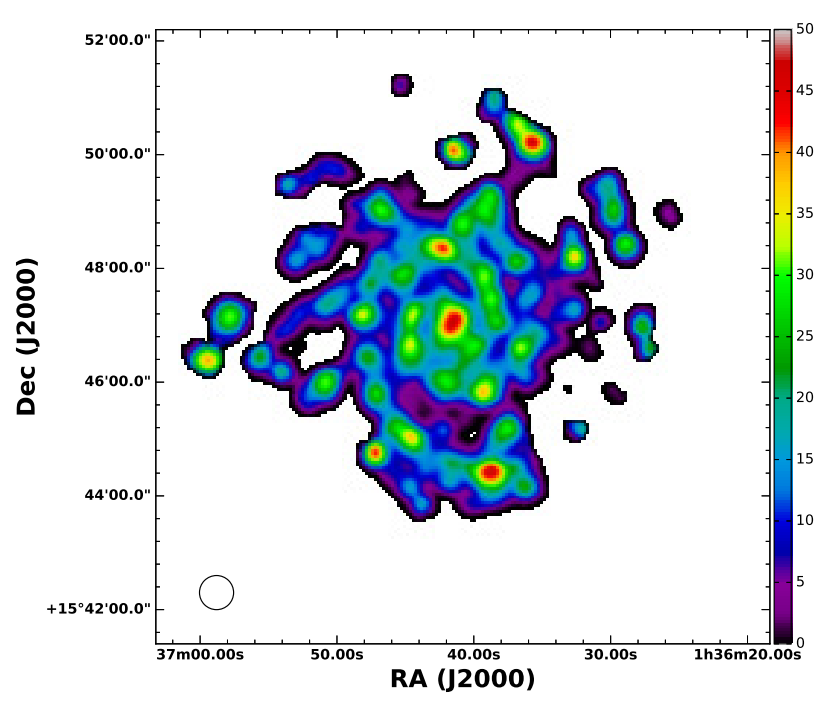

Fig. 11. Map of the thermal fraction at $3.1 \mathrm{GHz}$, computed from the total intensity and thermal images shown in Figs. 6 and 10 (left). Colour scale is in percent.

of the galaxy, we observe the usual $\mathrm{H} \alpha$ cut-off but extended UV emission still exists. There the SFR is so low that the small clusters do not contain massive stars and thus there is neither $\mathrm{H} \alpha$ nor radio emission (Pflamm-Altenburg \& Kroupa 2008). The extended northern spiral arm is seen in both $\mathrm{H} \alpha$ and UV emission but is much weaker compared to the rest of the galaxy.

Our observations indicate that the IGIMF in the central region is steeper compared to the rest of the galaxy. NGC 628 is an ideal galaxy to apply an IGIMF model with radial variation.

If there is no significant population of $\mathrm{O}$ stars, one cannot expect supernovae to inject fresh CREs. Given the nature of diffusion without fresh injection, a region of smooth radio continuum emission would be expected after sufficient time. The remaining cosmic ray electrons from the previous star formation period would have aged and diffused around the central region, creating a flat gradient and a steep spectrum in these regions. This explanation would require some CREs present to emit synchrotron emission and therefore that the CRE lifetime to be greater than the lifetime of $\mathrm{O}$ stars. Using the total magnetic field strengths found in Sect. 4.4 of $8-10 \mu \mathrm{G}$ for the central region we find the
CRE lifetime to be between 20-30 Myr. This is found to be several times greater compared to stellar evolution models of $\mathrm{O}$ stars e.g. found in Weidner \& Vink (2010) where a $120 M_{\odot}$ O type star can evolve to a carbon-rich Wolf-Rayet star in $3 \mathrm{Myr}$, one evolutionary stage before going supernovae. For a lower mass of $20 M_{\odot}$ it would $9 \mathrm{Myr}$ to reach the red super giant phase, again one evolutionary stage before going supernovae.

Low-frequency observations will greatly help along with CRE modelling with time-dependent injection profiles. This is a topic of further investigation.

\subsection{Magnetic field strength of NGC 628}

The total magnetic field strength of NGC 628 can be determined from the nonthermal emission by assuming equipartition between the energy densities of cosmic rays and magnetic field, using the revised formula of Beck \& Krause (2005). The total magnetic field strength scales with the synchrotron intensity $I_{\text {syn }}$ as:

$B_{\mathrm{tot}, \perp}=\left(I_{\mathrm{syn}} /\left(K_{0}+1\right) L\right)^{1 /\left(3-\alpha_{\mathrm{n}}\right)}$

where $B_{\text {tot, } \perp}$ is the strength of the total field perpendicular to the line of sight. Further assumptions required are the synchrotron spectral index of $\alpha_{\mathrm{n}}=-1.0$ and the effective path length through the source of $L=1000 \mathrm{pc} / \cos i \simeq 1007 \mathrm{pc}$ where $i$ is the inclination of the galaxy. We also assumed that the polarised emission emerges from ordered fields in the galaxy plane. The adopted ratio of CR proton to electron number densities of $K_{0}=100$ is a reasonable assumption in the star-forming regions in the disk (Bell 1978). Realistic uncertainties in $L$ and $K_{0}$ of a factor of about two would effect the result only by about $20 \%$. The effect of adjusting $\alpha$ to between -0.7 and -0.9 produces an error of less than $5 \%$ in magnetic field strength. Using these assumptions, we created an image of the total magnetic field in NGC 628 from the nonthermal map (Fig. 10, right) which is shown in Fig. 12.

The mean total magnetic field strength is around $9 \mu \mathrm{G}$ and $11-12 \mu \mathrm{G}$ in the spiral arm regions (Fig. 12). The largest and brightest star-forming complexes seen across the spiral arms have a total magnetic field strength around $14 \mu \mathrm{G}$, with a maximum strength greater than $15 \mu \mathrm{G}$ in the star-forming complex in the north. In the extended disk of the galaxy (a galactic centric radius of $\sim 8.2 \mathrm{kpc}$ ) we observe a magnetic field strength of approximately $8 \mu \mathrm{G}$. 


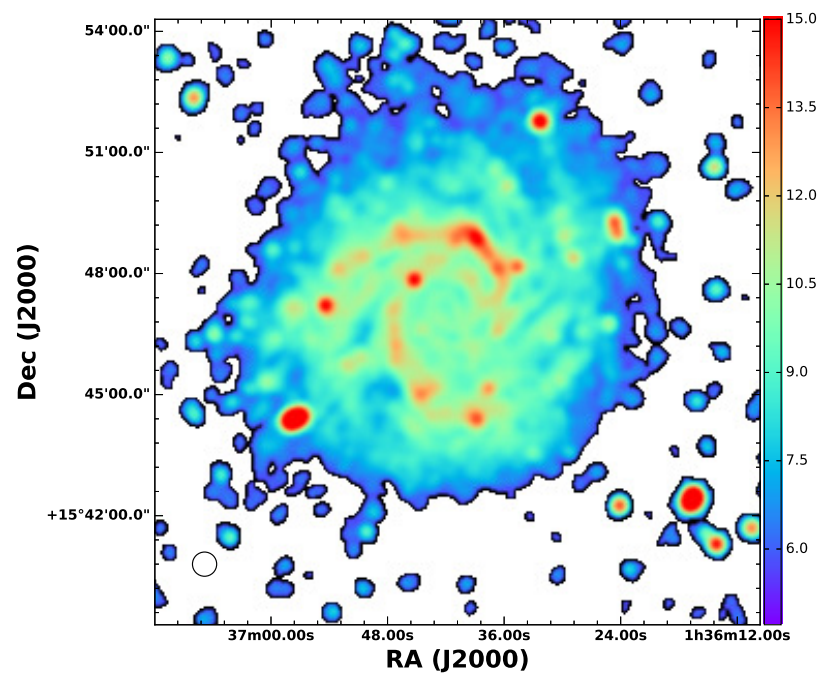

Fig. 12. Strength of the total magnetic field at $18^{\prime \prime}$ resolution, in units of $\mu \mathrm{G}$, determined by assuming energy equipartition.

NGC 628 has a similar magnetic field strength as NGC 6946 but a somewhat smaller field strength compared to IC 342 (Beck 2015), where the mean magnetic field strength is $14-15 \mu \mathrm{G}$ in the spiral arms. The main difference between NGC 628 and other galaxies in this respect is the central region, with a lower total magnetic field strength of $9-10 \mu \mathrm{G}$ compared to the central regions of NGC 6946 (about $25 \mu \mathrm{G}$; Beck 2007) and of IC 342 (about $30 \mu \mathrm{G}$; Beck 2015).

This method of calculating the total magnetic field strength most likely causes an underestimation of the magnetic field strength especially in the centre of the galaxy and extended disk due to the uncertainty of $K_{0}$ caused by ageing of CREs propagating into regions of low star formation. Ideally, low-frequency observations would be far more useful in determining the magnetic field strengths, not being contaminated by thermal emission (Mulcahy et al. 2014).

\subsection{Polarised intensity}

Images of linearly polarised intensity with overlaid $\boldsymbol{B}$-vectors at $10^{\prime \prime}$ and $18^{\prime \prime}$ resolutions are shown in the lower panels of Figs. 5 and 6. These maps were obtained by averaging $Q$ and $U$ over all channels, without correction for the effects of Faraday rotation between the channels.

Ordered magnetic fields are traced by polarised synchrotron emission and form spiral patterns in nearly every galaxy (Beck 2016), even in flocculent (Soida et al. 2002) and ring galaxies (Chyży \& Buta 2008). In NGC 628, three polarisation arms are prominent and are labelled in Fig. 5. These arms resemble the arms observed in IC 342 (Krause 1993; Beck 2015) and NGC 6946 (Beck 2007).

The first and most prominent polarisation spiral arm (arm 1) runs counter-clockwise, starting along the large $\mathrm{H}_{\text {II }}$ regions in the north-west, which are seen in total intensity (Fig. 4). This indicates that a fraction of the isotropic turbulent field is compressed or sheared and has become anisotropic turbulent. The region at the most northern point has the brightest polarised flux density and several interesting features makes it stand out from the rest of the galaxy. This region will be discussed in more detail in Sect. 5.5. Arm 1 spreads out into the inter-arm region of the galaxy in the south-east and continues to the south. The pitch angle of the polarisation vectors decreases along arm 1, from

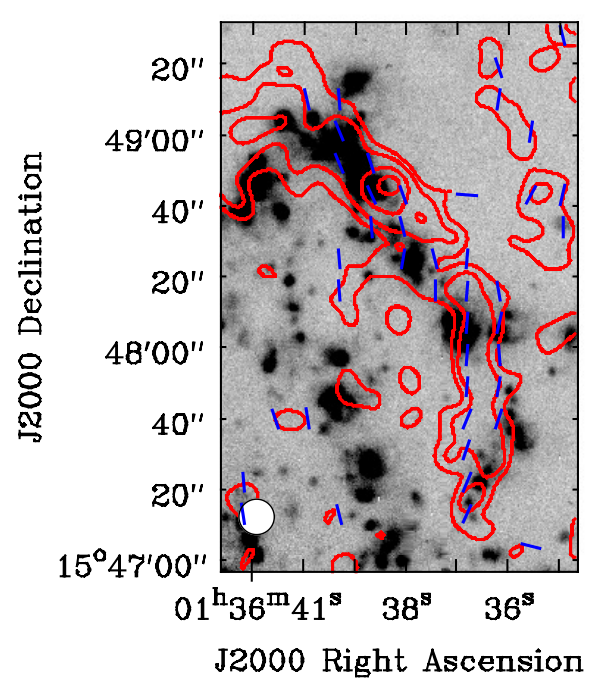

Fig. 13. Polarised emission following the largest $\mathrm{H}_{\text {II }}$ complexes in the galaxy. Polarised intensity and magnetic vectors are overlaid onto an $\mathrm{H} \alpha$ image (Dale et al. 2009). Contours of polarised intensity are at 9, 15,24 , and $30 \mu \mathrm{Jy} /$ beam. The resolution of the JVLA image at $10^{\prime \prime}$ is illustrated by the ellipse located in the bottom left corner.

about $50^{\circ}$ in the north-west to about $30^{\circ}$ in the east and south (see Table 8).

The spiral arm features in polarised intensity are located mostly between the optical arms, except in the inner (northwestern and northern) parts of the polarisation arm 1 (at approximately $\mathrm{RA}(\mathrm{J} 2000)=01^{\mathrm{h}} 36^{\mathrm{m}} 37-41^{\mathrm{s}} ; \operatorname{Dec}(\mathrm{J} 2000)=+15^{\circ} 47^{\prime}-$ $49^{\prime}$ ). Here, the polarised emission closely follows the $\mathrm{H}$ II complexes (Fig. 13). At small radii the polarised emission is located at the inner edge of the optical spiral arm and then crosses the ridge line delineated by $\mathrm{H}$ in regions to the outer edge of the arm. Finally, the polarisation coincides with a bright $\mathrm{H}$ iI complex.

The second and less prominent polarisation arm (arm 2) begins south-east of the centre of the galaxy and travels to the south-west. Arm 2 is narrower than arm 1. Another narrow arm (arm 3) begins in the south and continues into the extended disk in the north-west. The pitch angle of the polarisation vectors in arms 2 and 3 are large (about $45^{\circ}$ ) and do not vary significantly with increasing distance from the galaxy's centre (see Table 8). Arms 2 and 3 resemble magnetic arms ${ }^{4}$.

They are clearly offset from the optical arm (Figs. 5 and 14), narrow (about $1.1 \mathrm{kpc}$ ), and show a high degree of polarisation to the total intensity (on average $25 \%$ and up to $40 \%$ locally).

Both polarisation spiral arms are partly coincident with the H I gas (THINGS data, Walter et al. 2008), as shown in Fig. 15. Given that the Faraday depolarisation at this frequency is small (Fig. 20) and the inclination is low, we do not expect to detect any more polarisation arms. The polarisation arms may extend even further into the outer disk, especially the polarisation (magnetic) arm 3 in the north-west.

\footnotetext{
4 For polarised arms to be considered magnetic arms they must follow the required criteria as defined by Beck (2015):

- exist entirely in the interarm region of the galaxy, i.e. between the optical arms;

- be narrow $(\approx 1 \mathrm{kpc})$ and filamentary with an almost constant pitch angle;

- have a high degree of polarisation.
} 


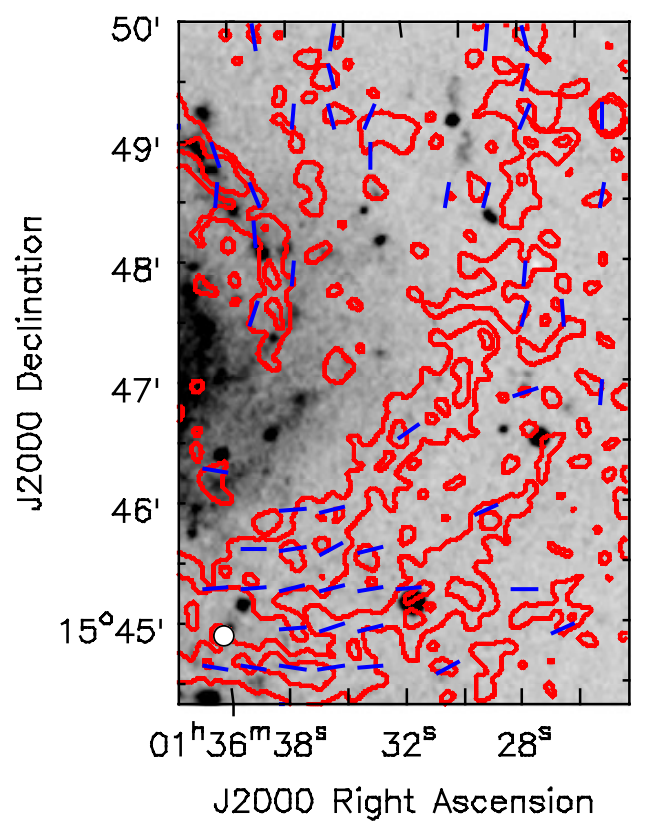

Fig. 14. Close-up of the magnetic arms located in an interarm region shown with polarised intensity and magnetic vectors overlaid onto an optical DSS image. Contours of polarised intensity are at 10 , $20 \mu \mathrm{Jy} / \mathrm{beam}$. The resolution of the JVLA image at $10^{\prime \prime}$ is illustrated by the ellipse located in the bottom left corner.

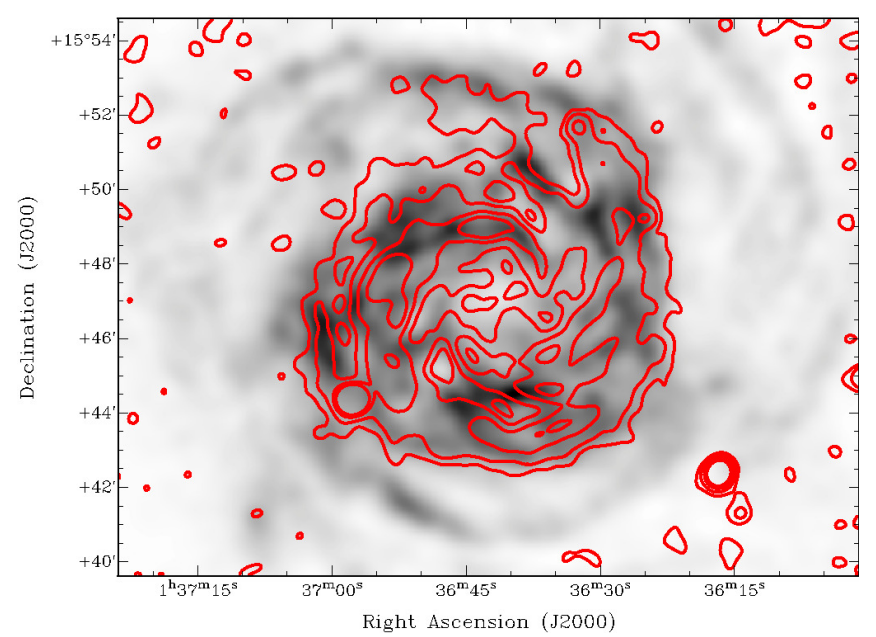

Fig. 15. Overlay of the polarised radio continuum emission onto the $\mathrm{H}_{\mathrm{I}}$ THINGS data, both smoothed to the same resolution of $30^{\prime \prime}$. Contours are shown at $40,80,120 \mu \mathrm{Jy} /$ beam.

We find degrees of polarisation for the inner polarisation arms between $10-25 \%$ at small radii, increasing to $50 \%$ at larger radii. This indicates an exceptionally ordered field. These degrees of polarisation are comparable to those at $1.5 \mathrm{GHz}$ measured by Heald et al. (2009) while we are able to detect significantly more polarisation, especially in the northern region of the galaxy, due to the smaller amount of depolarisation at $3.1 \mathrm{GHz}$.

A number of background sources are seen in polarisation, those closest to the phase centre are shown in Table 7. These sources are used to estimate the Faraday depth for the Galactic foreground in Sect. 5.3.

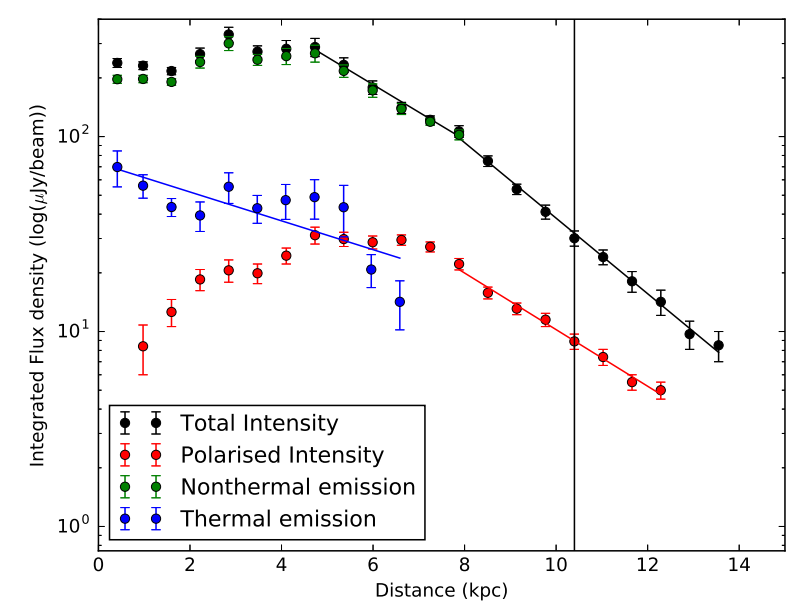

Fig. 16. Radial profile of NGC 628 for the total, nonthermal, thermal, and polarised radio intensities. The vertical line marks $R_{25}$.

\subsection{Radial scale lengths of the radio continuum emission}

The observed extent of disk emission is sensitivity limited. To characterise the emission along the disk we use the exponential scale length $l$, that is $I_{v} \propto \exp (-r / l)$ where $r$ is the galactocentric radius.

The radial profile of NGC 628 at $3.1 \mathrm{GHz}$ was taken from the total, nonthermal, thermal and polarised intensities averaged in concentric rings with the position angle of the major axis and the inclination of the galaxy taken into account, using the values from Table 1. Surrounding background point sources were removed by fitting and subtracting Gaussians before measuring the radial profile. Several background point sources located behind the inner disk were blanked out.

Fitting a single exponential profile is not possible, as a change in the slope of the total, nonthermal and polarised emission occurs at several points in the radial profile (Fig. 16). One such break in the slope occurs at approximately $6.5 \mathrm{kpc}$ which is approximately the radius where the SFR declines. This is the location where thermal emission and the injection of fresh CREs ends. Such a change of slope was a result in the cosmic ray electron propagation model of Mulcahy et al. (2016) for the galaxy M 51, using a realistic distribution of the injection of CREs. Beyond this radius, the total, nonthermal and polarised emission decrease exponentially.

It is unlikely that the lack of short spacings can cause the break in the radial profile of NGC 628. The largest angular scale of the JVLA at D configuration at $S$-band is $490^{\prime \prime}$, comparable to the angular size of NGC 628 (Table 1). To double check this, the JVLA map was smoothed and regridded to the same grid size as the Effelsberg $2.6 \mathrm{GHz}$ map and subtracted. No significant residual flux density was observed meaning that the JVLA observation was able to detect the same structures as the singledish observation, resulting in no missing flux density.

As there is a lack of emission at the centre of the galaxy in the total intensity, nonthermal, and polarised intensity maps, we were not able to fit an exponential function from the centre of the galaxy. Two separate exponential functions were fitted to the total intensity radial profile, inner $(4-6 \mathrm{kpc})$ and outer disk $(\geq 6.5 \mathrm{kpc})$ for both images:

$I(R)= \begin{cases}I_{4} \exp \left(-r / l_{\text {inner }}\right) & 4 \mathrm{kpc} \leq r \leq 6.5 \mathrm{kpc} \\ I_{6.5} \exp \left(-r / l_{\text {outer }}\right) & r \geq 6.5 \mathrm{kpc} .\end{cases}$ 
Table 6. Scale lengths of the inner and outer disk of NGC 628.

\begin{tabular}{lcc}
\hline \hline & $l_{\text {inner }}(\mathrm{kpc})$ & $l_{\text {outer }}(\mathrm{kpc})$ \\
\hline Total intensity & $3.1 \pm 0.2$ & $2.24 \pm 0.1$ \\
Polarised intensity & - & $3.0 \pm 0.1$ \\
Thermal intensity & $5.8 \pm 1.2$ & - \\
\hline
\end{tabular}

As $l_{\text {inner }}$ cannot be fit to the polarised intensity radial profile, only $l_{\text {outer }}$ was fitted. The thermal emission profile extracted from $\mathrm{H}_{\text {II }}$ displays an overall exponential decrease,with an arm region enhancement observed between 3 and $5 \mathrm{kpc}$. Here we fitted a single exponential function.

The radial profiles of the continuum emission for the total, nonthermal, thermal, and polarised intensity with the fitted functions are shown in Fig. 16. The obtained scale lengths for the inner and outer parts of the galaxy are given in Table 6 .

Many galaxies studied possess a radial profile where the maximum flux density is observed at the centre of the galaxy and decreases exponentially usually displaying arm and inter-arm features. Such galaxies include M 33 (Tabatabaei et al. 2007b), M 51 (Mulcahy et al. 2014), IC 342 (Beck 2015), and M 101 (Berkhuijsen et al. 2016).

In contrast, NGC 628 is much more complicated, indicating that a continuous injection of CREs, especially in the central region of the galaxy, is not valid. The total, nonthermal, and polarised emission show a minimum in the centre of the galaxy, peaking at around $3-4 \mathrm{kpc}$, the polarised emission peaking furthest out at $4 \mathrm{kpc}$. NGC 628 is unique with this absence of a bright central region and, as explained in Sect. 4.3, the CRE population is not sufficient to produce significant synchrotron emission.

\section{Observing the vertical magnetic field of NGC 628}

RM synthesis (Brentjens \& de Bruyn 2005; Heald 2009) allows us to measure Faraday rotation and investigate vertical magnetic fields. The resulting Faraday map sheds light on phenomena such as Parker loops and $\mathrm{H}_{\mathrm{I}}$ holes which shall now be described.

\subsection{RM synthesis}

When linearly polarised electromagnetic radiation passes through a magnetic-ionic medium, the plane of polarisation will rotate in a process known as Faraday rotation. When the polarised emission from a background source passes through a medium that is non-emitting (this type of medium is called a Faraday screen) or if Faraday rotation within the emitting medium is small (a Faraday thin medium), then the plane of polarisation will rotate by the following amount in radians:

$\Delta \chi=\mathrm{RM} \lambda^{2}$

where $\lambda$ is the wavelength of the polarised emission and RM is the rotation measure whose unit is $\mathrm{rad} \mathrm{m}^{-2}$ and is defined as the slope of the polarisation angle versus $\lambda^{2}$. For more general situations and in situations when regions with more than one rotation measure within the telescope beam are present, rotation measure is replaced with the quantity "Faraday depth" $\phi$. Faraday depth is defined as:

$\phi=0.812 \int_{\text {source }}^{\text {telescope }} n_{\mathrm{e}}(l) B_{\|}(l) \mathrm{d} l$.

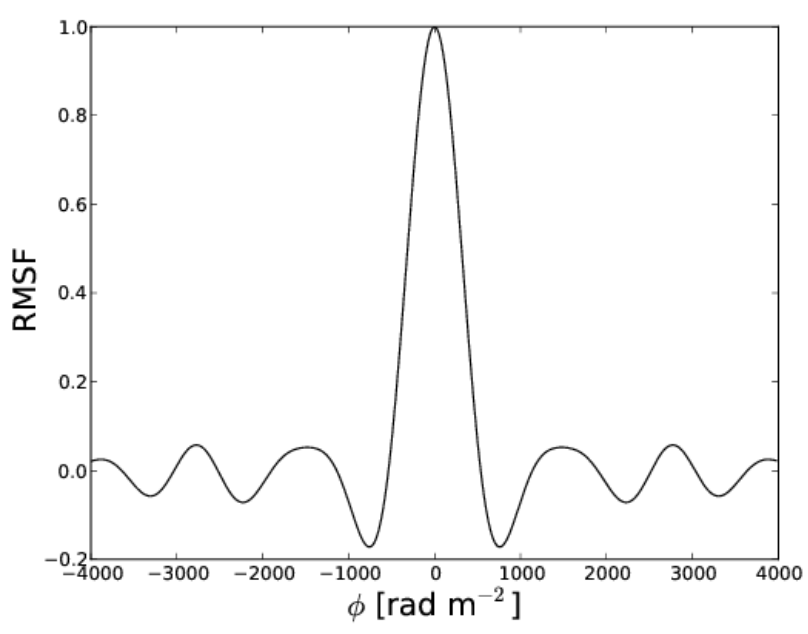

Fig. 17. RMSF of the remaining unflagged data channels $(2.6-3.6 \mathrm{GHz})$. Note due to the continuous bandwidth, the sidelobes of the RMSF are relatively low. The first sidelobe is approximately $20 \%$ whereas it was $78 \%$ for WSRT SINGS (Heald et al. 2009).

Here $n_{\mathrm{e}}(l)$ is the thermal electron density in $\mathrm{cm}^{-3}, B_{\|}$is magnetic field strength along the line of sight in $\mu \mathrm{G}$, and $\mathrm{d} l$ is the path length in parsecs.

RM synthesis is a novel approach (Brentjens \& de Bruyn 2005) to extract the distribution of Faraday depths ("Faraday spectrum") of a source through polarimetric data whose effectiveness depends on the $\lambda^{2}$ coverage of the observation. With the JVLA's large bandwidth, RM synthesis is an ideal tool to investigate the nature of the vertical magnetic field of NGC 628.

The data were averaged in frequency channels in bins of 8 , resulting in 64 channels with a bandwidth of $16 \mathrm{MHz}$ each. The maximum Faraday depth for this channel width at $2.6 \mathrm{GHz}$ is $1477 \mathrm{rad} \mathrm{m}^{-2}$, much larger than what is expected for NGC 628 . With the coverage in $\lambda^{2}$, the maximum theoretical Faraday resolution is $570 \mathrm{rad} \mathrm{m}^{-2}$, while the maximum observable scale in the Faraday spectrum is $458 \mathrm{rad} \mathrm{m}^{-2}$. The resulting RM spread function (RMSF) of this $\lambda^{2}$ coverage is shown in Fig. 17.

We created channel images with natural weighting at a resolution of $18^{\prime \prime}$, using the mosaic option of the CASA clean task rather than mosaicing all the fields manually after cleaning. These images then had the primary beam correction applied. Each channel was visually inspected for RFI and one channel was deemed unusable. Therefore the final number of channel images used for RM synthesis was 63.

RM synthesis and RM clean (Heald et al. 2009) were both applied to the data using the pyrmsynth software ${ }^{5}$. A search for large Faraday depths in NGC 628 was performed over the range of $-2 \times 10^{5} \mathrm{rad} \mathrm{m}^{-2}$ to $+2 \times 10^{5} \mathrm{rad} \mathrm{m}^{-2}$ with a coarse $\phi$ sampling of $1000 \mathrm{rad} \mathrm{m}^{-2}$. No such polarised emission was detected at very large Faraday depths. Hence, RM synthesis was performed only in the range $-3000 \mathrm{rad} \mathrm{m}^{-2}$ to $+3000 \mathrm{rad} \mathrm{m}^{-2}$ with a sampling of $25 \mathrm{rad} \mathrm{m}^{-2}$. The rms noise measured in the Stokes $Q$ and $U$ cubes is 3.6 and $4.2 \mu \mathrm{Jy} /$ beam, respectively.

The maximum Faraday depth $\left(\phi_{\max }\right)$ in the Faraday spectrum at each pixel of the map was measured by fitting a parabola using the three maximum points at the observed peak in the Faraday spectrum. Only polarised flux densities above a $6 \sigma$ level of $21.6 \mu \mathrm{Jy} /$ beam were used. The Milky Way contribution (see Sect. 5.3) of $-34 \mathrm{rad} \mathrm{m}^{-2}$ was subtracted from this Faraday depth.

5 https://mrbell.github.io/pyrmsynth/ 

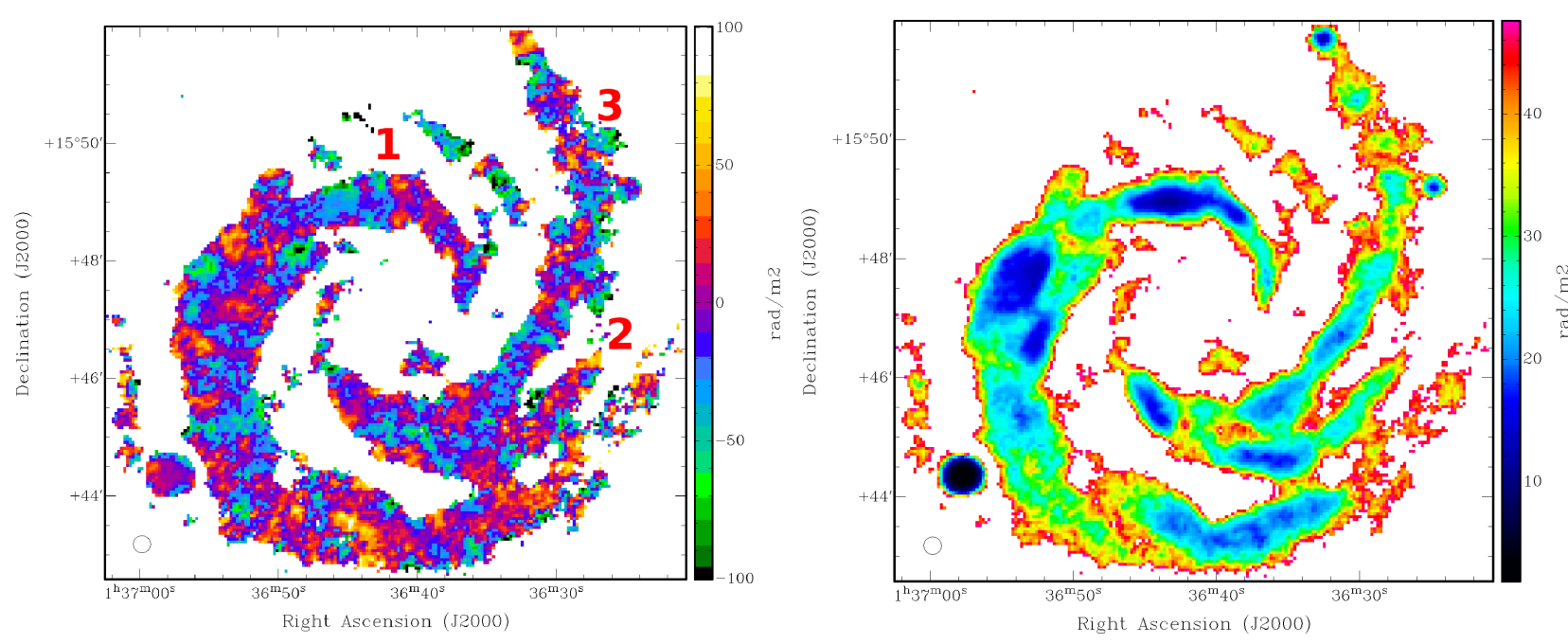

Fig. 18. Maps of the maximum fitted Faraday depth $\phi_{\max }(l e f t)$ and the corresponding Faraday depth error $\sigma_{\phi}($ right $)$ at a resolution of $18^{\prime \prime}$ marked by an ellipse in the bottom left. Both quantities are measured in units of $\mathrm{rad} \mathrm{m}^{-2}$. The polarised arms defined in Fig. 5 are shown here marked in red.

The corresponding $Q$ and $U$ values were found from the nearest pixel to that of the measured maximum Faraday depth and were used to find the intrinsic polarisation angle (corrected for Faraday rotation via RM synthesis) by:

$\chi_{0}=\frac{1}{2} \arctan \left(\frac{U\left(\phi_{\max }\right)}{Q\left(\phi_{\max }\right)}\right)$

The magnetic field orientation is obtained by rotating the polarisation angle by $90^{\circ}$.

The polarised intensity image was created using the AIPS task "COMB" with the "POLC" options which takes into account the bias polarised intensity caused by noise in Stokes $Q$ and $U$. We used the average rms noise of the $Q$ and $U$ maps to correct for this bias (see Eq. (1)). The polarised intensity map is very similar to the channel averaged image (Fig. 6). This is due to the fact that Faraday rotation between the frequency channels at this frequency is small. The only significant difference is the slightly more extended emission seen in the south east of the galaxy.

The errors in Faraday depth and intrinsic polarisation angle were determined by:

$\Delta \phi=\frac{\phi_{\mathrm{rmsf}}}{2 \mathrm{SR}}$

and

$\Delta \chi_{0}=\frac{1 \mathrm{rad}}{2 \mathrm{SR}}$

where $\phi_{\text {rmsf }}$ is the FWHM of the RMSF and SR is the signal-tonoise ratio of the polarised intensity.

The maps of maximum Faraday depth and associated error are shown in Fig. 18.

RM synthesis has been performed on NGC 628 previously at 18 and $22 \mathrm{~cm}$ wavelengths with the WSRT by Heald et al. (2009) with a Faraday depth resolution of $144 \mathrm{rad} \mathrm{m}^{-2}$, with the dominant Faraday depth component centred on $-30 \mathrm{rad} \mathrm{m}^{-2}$. While their Faraday resolution was superior compared to the present work, the sidelobe level was much higher due to the gap between the 18 and $22 \mathrm{~cm}$ bands. Additionally, this work has much better angular resolution and sensitivity and therefore we are able to resolve different features in our Faraday depth map (Fig. 18 left).

The main Faraday depth component observed in NGC 628 varies between $+100 \mathrm{rad} \mathrm{m}^{-2}$ and $-110 \mathrm{rad} \mathrm{m}^{-2}$. The mean Faraday depth is $-8 \mathrm{rad} \mathrm{m}^{-2}$ with a standard deviation of $30 \mathrm{rad} \mathrm{m}^{-2}$. This agrees with the dominant Faraday depth component at $30 \mathrm{rad} \mathrm{m}^{-2}$ observed by Heald et al. (2009). The standard deviation of $30 \mathrm{rad} \mathrm{m}^{-2}$ contains real structure that is likely to be complex. The presence of a vertical magnetic field and the large scale height of cosmic-ray electrons tend to make the standard deviation of the Faraday dispersion function larger (Ideguchi et al. 2014).

We see an interesting and striking periodic pattern alternating between negative and positive Faraday depth values in the northern and eastern parts of arm 1. In the southern part of arm 1 no such pattern is seen, and the Faraday depths are mostly positive. Arms 2 and 3 neither show such a periodic pattern nor any obvious large-scale pattern in Faraday depth but has more negative Faraday depths than the southern part of arm 1 (see Sects. 5.8 and 6.2 for details).

Braun et al. (2010) detected secondary components for NGC 628 at $\phi=-213$ and $+145 \mathrm{rad} \mathrm{m}^{-2}$ in addition to other mildly inclined galaxies such as NGC 6946 and M51. These secondary components may originate from polarisation at the far side of the midplane, becoming more Faraday rotated when passing through the midplane, resulting in larger values of Faraday depths, approximately $\pm 200 \mathrm{rad} \mathrm{m}^{-2}$. Mao et al. (2015) searched for these secondary components in M51 with the JVLA at $L$ band and did not detect any significant polarisation coinciding with these secondary components. We have searched the Faraday cube at $18^{\prime \prime}$ and could not find indications in the Faraday spectra of these secondary components. It should be noted, however, that the Faraday depth resolution for this observation is not enough to fully resolve the main component from the secondary components.

\subsection{Magnetic field order}

The nonthermal polarisation degree $p_{\mathrm{n}}$ is a measure of the ratio $q$ of the field strength of the ordered field in the sky plane and the random field, named the degree of order of the field, $q=B_{\mathrm{reg}} / B_{\mathrm{ran}}$ (Beck 2007). The maximum possible degree of 

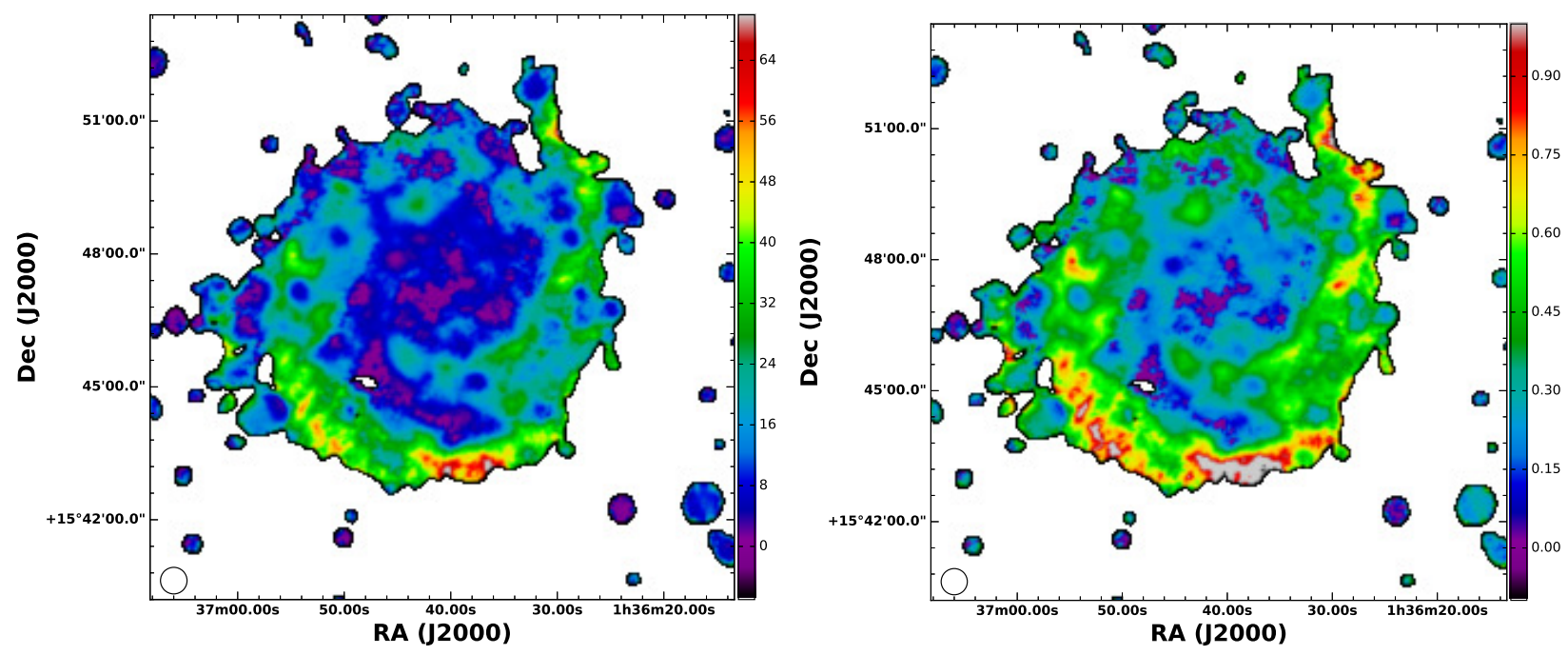

Fig. 19. Nonthermal polarisation degree (left) and the degree of field order $q$ (right), the ratio of the ordered field strength in the sky plane to the isotropic random field strength, derived from the fractional polarisation of the nonthermal intensity (Fig. 10, right) The colour scale is in percent (left) and degree of field order (right).

polarisation is given by $p_{0}=\left(3-3 \alpha_{\mathrm{n}}\right) /\left(5-3 \alpha_{\mathrm{n}}\right)$ where $\alpha_{\mathrm{n}}$ is the nonthermal spectral index taken here as -1.0 .

In case of equipartition between the energy densities of magnetic field and cosmic rays (Beck 2007):

$$
\begin{aligned}
& \frac{p_{\mathrm{n}}}{p_{0}}=\frac{q^{2}}{\left(q^{2}+\frac{1}{3}\right)} \\
& q \simeq\left(\frac{\left(p_{\mathrm{n}} / p_{0}\right)}{\left[2\left(1-\left(p_{\mathrm{n}} / p_{0}\right)\right)\right]}\right)^{0.5} .
\end{aligned}
$$

Figure 19 shows the nonthermal polarisation degree (left) and the degree of magnetic field order (right) for NGC 628 in the case of equipartition which was derived from the polarised intensity map found from RM synthesis (Fig. 18, left) and the nonthermal map (Fig. 10, right). $q$ is almost zero in the optical spiral arms, meaning that depolarisation is enhanced in the spiral arms. $q$ increases toward the outer radii, specifically in the inter-arm regions where $q$ reaches 0.8 where depolarisation is low. An isolated region with a maximum $q$ of 0.56 exists in the northern part of polarisation arm 1 and coincides with the region of the most intense polarised emission, surrounded by $q$ values of around 0.2. This region will be discussed in further detail in Sect. 5.5.

\subsection{Estimate of the Galactic foreground in the direction of NGC 628}

Based on three polarised sources detected in the NGC 628 field, Heald et al. (2009) estimated that the likely Galactic foreground Faraday depth is about $-34 \pm 2 \mathrm{rad} \mathrm{m}^{-2}$. In our NGC 628 field we detect seven polarised sources, four of which are not directly located behind NGC 628. Table 7 shows the locations, Faraday depths, and polarised intensities of these discrete sources. The average of the Faraday depth of these four sources gives us a likely value of $-20 \pm 14 \mathrm{rad} \mathrm{m}^{-2}$. It is possible that the Faraday depth of these sources used is still affected by NGC 628. These sources are found in the range of $16-23 \mathrm{kpc}$ from the centre of NGC 628. Han et al. (1998) found through RM data of background sources that in M 31 the regular magnetic field probably extends to a radius of $25 \mathrm{kpc}$.
A Galactic foreground Faraday depth of $-20 \pm 14 \mathrm{rad} \mathrm{m}^{-2}$ is comparable to that of Heald et al. (2009). As the Faraday depth resolution in Heald et al. (2009) was superior to that of our work, $-34 \mathrm{rad} \mathrm{m}^{-2}$ was taken as the likely value when subtracting the Galactic foreground contamination. This also highlights the importance of $L$-band observations to achieve better Faraday depth resolution and thus ensure a more accurate removal of the Faraday rotation caused by the Galactic foreground.

\subsection{Depolarisation within S-band}

Faraday depolarisation is an important tool in retrieving information on the density of ionised gas, the strength of the ordered and turbulent field components, and the typical length scale (or integral scale) of turbulent magnetic fields. Significant depolarisation is to be expected for NGC 628, as Heald et al. (2009) observed little to no polarised emission in the northern regions of the galaxy.

In order to get an estimation of the Faraday depolarisation we define the ratio DP (Beck 2007) by:

$\mathrm{DP}=\left(\frac{\mathrm{PI}_{2.87 \mathrm{GHz}}}{\mathrm{PI}_{3.43 \mathrm{GHz}}}\right) \times\left(\frac{3.43 \mathrm{GHz}}{2.87 \mathrm{GHz}}\right)^{\alpha_{\mathrm{n}}}$

where $\alpha_{\mathrm{n}}=-1.0$ is the nonthermal spectral index and is assumed to be constant across the entire galaxy. $\mathrm{DP}=1$ signifies no depolarisation and $\mathrm{DP}=0$ means total depolarisation. We image two sections of the band which have the same $\lambda^{2}$ coverage. These two bands have central frequencies of 2.87 and $3.43 \mathrm{GHz}$. The computed depolarisation map is shown in Fig. 20. We observe depolarisation ratios between 0.6 and 1.4 but the largest ratios are uncertain due to low signal-to-noise ratios. DP is about 0.9 on average and 0.7 in star-forming regions. Varying $\alpha_{\mathrm{n}}$ by 0.2 changes the DP ratio by 0.03 , likewise a $10 \%$ difference in polarised intensity changes DP by 0.1 .

Generally, lower DP ratios, i.e. stronger depolarisation, are seen in the northern parts of the of the galaxy, located around the major axis $\left(\mathrm{PA}=20^{\circ}\right)$. This is consistent with the findings of Heald et al. (2009) and Braun et al. (2010) that for many galaxies Faraday depolarisation at $\lambda 20 \mathrm{~cm}$ is asymmetric along the 
Table 7. Discrete polarised sources detected in the NGC 628 field, uncorrected for Faraday rotation caused by the Galactic foreground.

\begin{tabular}{cccccccc}
\hline \hline $\begin{array}{c}\text { RA } \\
(\mathrm{h} \mathrm{m} \mathrm{s})\end{array}$ & $\begin{array}{c}\text { Dec } \\
\left({ }^{\circ}{ }^{\prime \prime}\right)\end{array}$ & $\begin{array}{c}\text { Galactocentric radius } \\
(\mathrm{kpc})\end{array}$ & $\begin{array}{c}\text { Peak flux density } \\
(\mu \mathrm{Jy} / \mathrm{beam})\end{array}$ & $\begin{array}{c}\phi \\
\left(\mathrm{rad} / \mathrm{m}^{2}\right)\end{array}$ & $\begin{array}{c}\Delta \phi \\
\left(\mathrm{rad} / \mathrm{m}^{2}\right)\end{array}$ & $\begin{array}{c}\mathrm{PA} \\
\left({ }^{\circ}\right)\end{array}$ & $\begin{array}{c}\Delta \mathrm{PA} \\
\left({ }^{\circ}\right)\end{array}$ \\
\hline $\mathbf{0 1 ~ 3 6 ~ 0 0}$ & $\mathbf{+ 1 5 4 4 5 8}$ & $\mathbf{2 1 . 4 2}$ & $\mathbf{8 7}$ & $\mathbf{- 1 3 . 7}$ & $\mathbf{1 1 . 6}$ & $\mathbf{1 2 . 7}$ & $\mathbf{1 . 1}$ \\
$\mathbf{0 1 ~ 3 6 ~ 1 4 ~}$ & $\mathbf{+ 1 5 4 1 ~ 1 6}$ & $\mathbf{1 8 . 3 7}$ & $\mathbf{6 9}$ & $\mathbf{- 6 . 5}$ & $\mathbf{1 4 . 7}$ & $\mathbf{- 6 8 . 9}$ & $\mathbf{1 . 4}$ \\
$\mathbf{0 1 ~ 3 6 ~ 1 6 ~}$ & $\mathbf{+ 1 5 4 2 ~ 2 5}$ & $\mathbf{1 6 . 1 4}$ & $\mathbf{4 0 5}$ & $\mathbf{- 3 9 . 1}$ & $\mathbf{2 . 5}$ & $\mathbf{- 5 2 . 0}$ & $\mathbf{0 . 3}$ \\
013624 & +154913 & 9.80 & 67 & -49.5 & 15.5 & -86.8 & 1.5 \\
013632 & +155143 & 10.80 & 85 & +6.1 & 11.49 & 76.0 & 1.2 \\
013657 & +154422 & 9.87 & 532 & -34.5 & 1.9 & -88.9 & 0.2 \\
$\mathbf{0 1 3 7 ~ 2 8}$ & $\mathbf{+ 1 5 4 6 ~ 1 8}$ & $\mathbf{2 3 . 3 4}$ & $\mathbf{1 4 0}$ & $\mathbf{- 2 1 . 8}$ & $\mathbf{7 . 3}$ & $\mathbf{4 0 . 2}$ & $\mathbf{0 . 7}$ \\
\hline
\end{tabular}

Notes. Sources used to calculate the Galactic foreground are marked in bold.

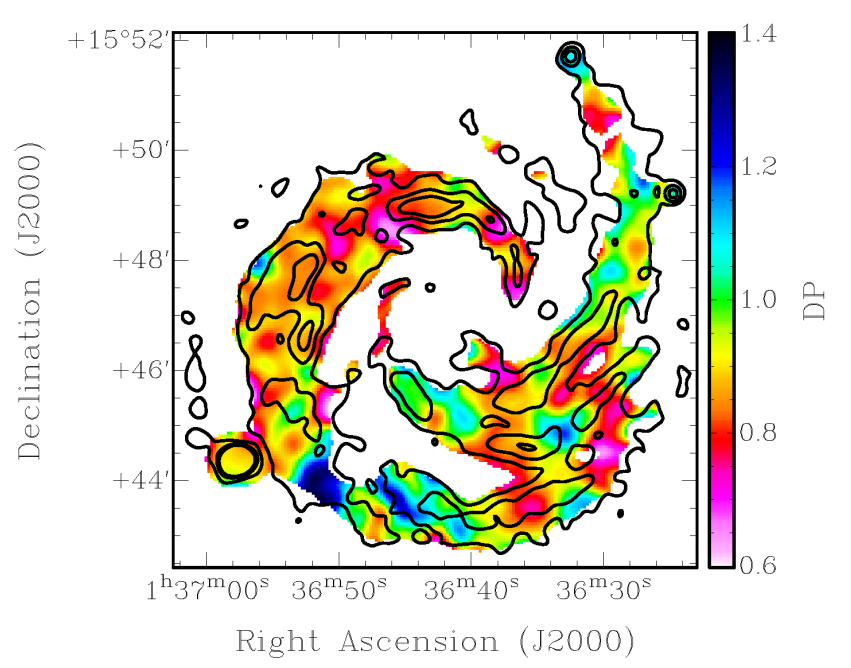

Fig. 20. Map of the Faraday depolarisation ratio of NGC 628 across the $S$-band at $31^{\prime \prime}$ resolution, with contours of polarised intensity at 20,40 , $60 \mu \mathrm{Jy} /$ beam.

major axis of the projected galaxy disk in the sky plane, caused by the large-scale halo field. This results in less polarised emission on the kinematically receding side of the major axis. Other regions with low DP are located where star formation and thus $B_{\text {ran }}$ and thermal electron density are greatest.

There are two main mechanisms that cause depolarisation. The first, differential Faraday rotation, arises when relativistic and thermal electrons occupy the same region of the magnetised medium. The degree of polarisation $p$ depends on the observable Faraday rotation measure RM and $\lambda$ as (Burn 1966; Sokoloff et al. 1998):

$p=p_{0} \frac{\sin \left|2 \mathrm{RM} \lambda^{2}\right|}{\left|2 \mathrm{RM} \lambda^{2}\right|}$

where $p_{0}$ is the intrinsic degree of polarisation. The polarisation vanishes when $|\mathrm{RM}|=\mathrm{RM}_{0}$ where

$2 \mathrm{RM}_{0} \lambda^{2}=\pi n$

with $n=1,2, \ldots$ This causes depolarisation canals in the ISM as seen extensively in M 31 at $\lambda 20.5 \mathrm{~cm}$ (Shukurov \& Berkhuijsen 2003). For NGC 628 at $\lambda 9.6 \mathrm{~cm}$, we find $\mathrm{RM}_{0}=170 \mathrm{rad} \mathrm{m}^{-2}$ which is larger than the average Faraday depth observed in Fig. 18. Thus we expect little depolarisation due to differential Faraday rotation at this frequency. Indeed, no depolarisation canals can be seen in any of the polarisation maps.
The second mechanism that causes depolarisation, internal Faraday dispersion by turbulence in the magnetised ISM, is the most probable source of depolarisation in NGC 628 (Sokoloff et al. 1998):

$p=p_{0} \frac{1-\exp (-2 S)}{2 S}$

where $S=\sigma_{\mathrm{RM}}^{2} \lambda^{4} . \sigma_{\mathrm{RM}}$ is the dispersion in intrinsic rotation measure $\mathrm{RM}_{i} . \sigma_{\mathrm{RM}}$ of $43 \mathrm{rad} \mathrm{m}^{-2}$ is needed to produce $\mathrm{DP}=0.9$ between $\lambda 0.087 \mathrm{~m}$ and $\lambda 0.104 \mathrm{~m}$.

Internal Faraday dispersion is a consequence of the turbulent ISM and can be written as $\sigma_{\mathrm{RM}}=0.812 n_{\mathrm{e}} \sqrt{1 / 3} B_{\mathrm{ran}} \sqrt{L d / f}$ (Beck 2016) where $n_{\mathrm{e}}$ is the average thermal electron density of the diffuse ionised gas along the line of sight (in $\mathrm{cm}^{-3}$ ), $B_{\text {ran }}$ the random field strength (in $\mu \mathrm{G}$ ), $L$ the path length through the thermal gas (in pc), $d$ is the turbulent scale (in pc), and $f$ the filling factor of the Faraday-rotating gas. Standard values found in NGC 6946 (Beck 2007) of $n_{\mathrm{e}}=0.03 \mathrm{~cm}^{-3}, B_{\text {ran }}=10 \mu \mathrm{G}, L=$ $1000 \mathrm{pc}, d=50 \mathrm{pc}$, and $f=0.5$ yield $\sigma_{\mathrm{RM}} \simeq 44 \mathrm{rad} \mathrm{m}^{-2}$, as required for NGC 628. For the star-forming regions, increasing the thermal electron density or the random field strength by a factor of two yields the required value of $\mathrm{DP}=0.7$.

\subsection{A lone ordered magnetic field in NGC 628?}

The most intense polarised emission is seen in the northern part of polarisation arm 1 at $\operatorname{RA}(J 2000)=01^{\mathrm{h}} 36^{\mathrm{m}} 44^{\mathrm{s}}$, $\operatorname{Dec}(\mathbf{J} 2000)=+15^{\circ} 48^{\prime} 58^{\prime \prime}$. This region could be a Faraday window with low Faraday depolarisation (i.e. a high DP ratio). However, the $S$-band depolarisation map (Fig. 20) shows normal DP ratios of around 0.8 , similar to other regions in the galaxy and must then originate from truly ordered fields.

The region has a degree of magnetic field order $q$ of approximately 0.56 (Fig. 19). The degree of polarisation in this region has a mean of $19 \%$ and a maximum of $29 \%$. The immediate area around this particular region has degrees of polarisation of less than $10 \%$. While this is not especially high compared to the outer regions of the galaxy, it is quite high with respect to the immediate vicinity ( $q$ of 0.2 ). This region is also apparent and out of place with its small pitch angle of $25^{\circ}$ compared to its surroundings with a pitch angle of approximately $45^{\circ}$ (Fig. 24). This feature is unique as nothing similar has been detected so far in any other galaxy.

A compressed magnetic field due to substantial ram pressure caused by the motion of the galaxy through the intergalactic medium (IGM; like in the Virgo cluster, see Vollmer et al. 2007) does not seem to be a valid explanation as NGC 628 shows no signs of gas stripping in the outer parts; instead, it has a large 
Table 8. Average pitch angles of spiral arm structures of polarised intensity and magnetic pitch angles of the ordered field of NGC 628 .

\begin{tabular}{lrrrr}
\hline \hline Spiral feature & Azimuthal range $\left(^{\circ}\right)$ & Radial range $\left(^{\prime}\right)$ & Morphological pitch angle $\left(^{\circ}\right)$ & Magnetic pitch angle $\left(^{\circ}\right)$ \\
\hline 1A & $264-344$ & $0.22-1.04$ & $+25 \pm 2$ & $+50 \pm 8$ \\
1B & $310-402$ & $0.72-0.98$ & $+9 \pm 1$ & $+40 \pm 7$ \\
1C & $336-76$ & $0.66-1.38$ & $+20 \pm 2$ & $+32 \pm 10$ \\
1D & $114-210$ & $1.34-1.36$ & $+2 \pm 2$ & $+27 \pm 8$ \\
2 & $118-206$ & $0.26-1.18$ & $+30 \pm 3$ & $+42 \pm 8$ \\
3A & $162-244$ & $0.26-0.86$ & $+23 \pm 2$ & $+43 \pm 7$ \\
3B & $244-284$ & $0.86-1.42$ & $+24 \pm 2$ & $+43 \pm 7$ \\
3C & $284-324$ & $1.42-1.76$ & $+46 \pm 9$ \\
\hline
\end{tabular}

Notes. Note that the pitch angle is positive for a spiral that is winding outwards in the counter-clockwise sense.

intact H I disk, especially in the north where the polarised region is located.

The magnetic field in this region may be compressed due to an expanding superbubble caused by several supernovae. We consider this to be a plausible explanation, since tentative signs of gas expansion are present in the $\mathrm{H}_{\mathrm{I}}$ data from the THINGS survey. Although there are no previously catalogued Hi holes in this region (Bagetakos et al. 2011), a position velocity diagram (Fig. 22) of the region shows tentative kinematic signs of a bubble. This double-peaked kinematic signature corresponds to a depression in the local H i column density that has an elliptical morphology overlapping the extent of the polarised region. In fact, this region was identified as a potential hole during the preparation of the catalogue presented by Bagetakos et al. (2011), but was excluded from the final list due to the relatively low apparent expansion velocity $\left(\sim 20 \mathrm{~km} \mathrm{~s}^{-1}\right.$ as compared to the resolution of the data, $\sim 8 \mathrm{~km} \mathrm{~s}^{-1}$ ) combined with the fact that the expansion is not seen along the minor axis of the elliptical feature. The $\mathrm{H}_{\mathrm{I}}$ features are suggestive, but not conclusive, of an expanding bubble (Y. Bagetakos, priv. comm.). On the other hand, bright $\mathrm{H} \alpha$ features are seen around the periphery of the $\mathrm{H}$ I feature, which could indicate recent star formation induced by the compression of ISM material at the edges of an expanding feature. Taken all together, these features could be consistent with a barrel-shaped expansion taking place preferentially along the direction of the ordered magnetic field.

We conclude that the northern region hosts an exceptionally strong and ordered field. This region has a small Faraday depth of -5 to $-10 \mathrm{rad} / \mathrm{m}^{2}$, very similar to other regions of the galaxy and thus signifies that, while the field in the sky plane is highly ordered, the magnetic field in the line of sight is not particularly strong. We propose that the polarisation peak is caused by a strongly ordered magnetic field driven by an asymmetrically expanding $\mathrm{H}_{\mathrm{I}}$ bubble, and possibly accentuated by low turbulence due to the absence of recent star formation in the centre of the feature, evident from the lack of $\mathrm{H} \alpha$ and UV emission (Gil de Paz et al. 2007) as illustrated in Fig. 21. Observations at other frequencies could help to further constrain the cause of such an isolated, highly ordered magnetic field.

\subsection{H I holes}

The Faraday depth (FD) map (Fig. 18) was compared by eye to the $\mathrm{H}_{\mathrm{I}}$ hole catalogue of Bagetakos et al. (2011). A Faraday depth gradient like the one seen in NGC 6946 by Heald (2012) is detected, as we observe a region with a large Faraday depth feature, the largest one in NGC 628 coinciding with a H I hole (Fig. 23) with a Faraday depth gradient, with FD decreasing from $-20 \mathrm{rad} \mathrm{m}^{-2}$ at the western edge of the Hi hole

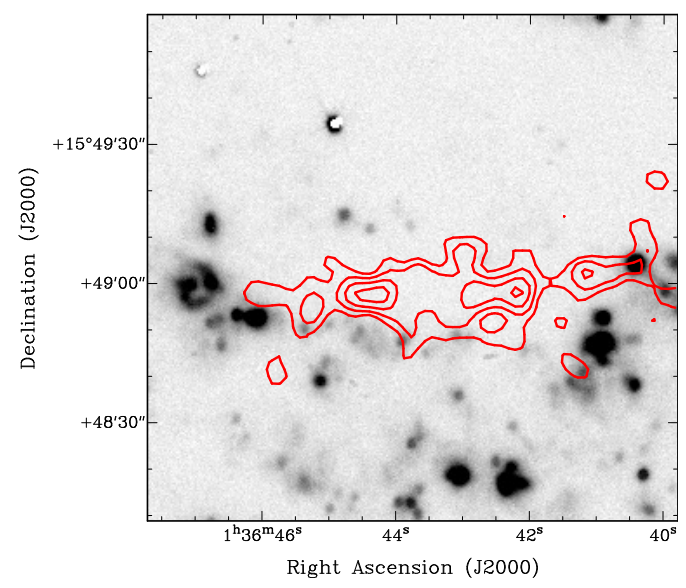

Fig. 21. Northern region of NGC 628 hosting an exceptionally strong and ordered magnetic field discussed in Sect. 5.5 shown with polarised intensity overlaid onto a $\mathrm{H} \alpha$ image (Dale et al. 2009). Contours are at $10,15,20,25 \mu \mathrm{Jy} /$ beam. Resolution is $7.5^{\prime \prime}$.

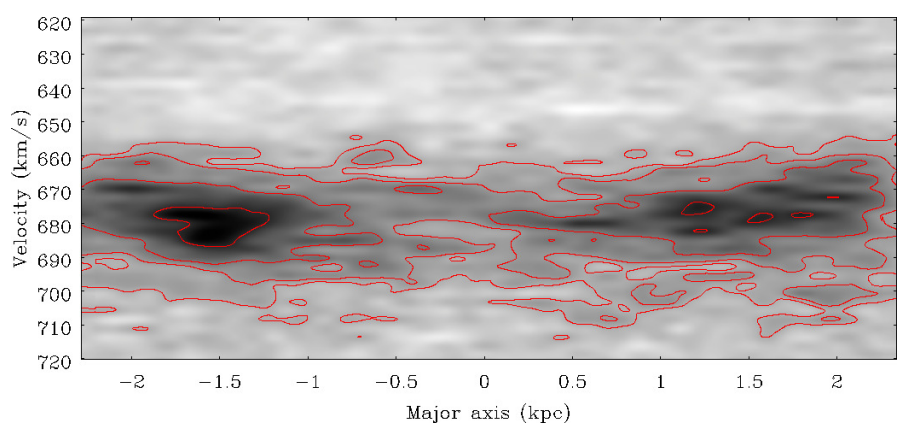

Fig. 22. H I position velocity diagram of a $1 \mathrm{D}$ slice through the region of a lone ordered magnetic field, as described in Sect. 5.5. The centre of this region is located at $0 \mathrm{kpc}$ and extends approximately from -1 to $1 \mathrm{kpc}$. Contours are at $3,5,8,14 \times \sigma(\sigma \simeq 5 \mu \mathrm{Jy} /$ beam $)$.

to $-130 \mathrm{rad} \mathrm{m}^{-2}$ at the eastern edge. This $\mathrm{H}$ i hole is located at $\mathrm{RA}(\mathrm{J} 2000)=01^{\mathrm{h}} 36^{\mathrm{m}} 48^{\mathrm{s}}, \operatorname{Dec}(\mathrm{J} 2000)=+15^{\circ} 48^{\prime} 06^{\prime \prime}$ with an expansion velocity of $7 \mathrm{~km} \mathrm{~s}^{-1}$ and a kinetic age of $50 \mathrm{Myr}$.

The age of an $\mathrm{H}$ i hole is crucial in order to observe any Faraday depth gradient, as it must be old enough to have gained a certain vertical offset but young enough so that vertical shear has not destroyed the Faraday signature. For a vertical shear of $\approx 15 \mathrm{~km} \mathrm{~s}^{-1} \mathrm{kpc}^{-1}$, similar to NGC 891 (Heald et al. 2006), the characteristic age is approximately $60 \mathrm{Myr}$. Therefore, this $\mathrm{H} \mathrm{I}$ hole has an ideal age to observe such a Faraday depth gradient caused by magnetic fields carried by hot gas motions originating 


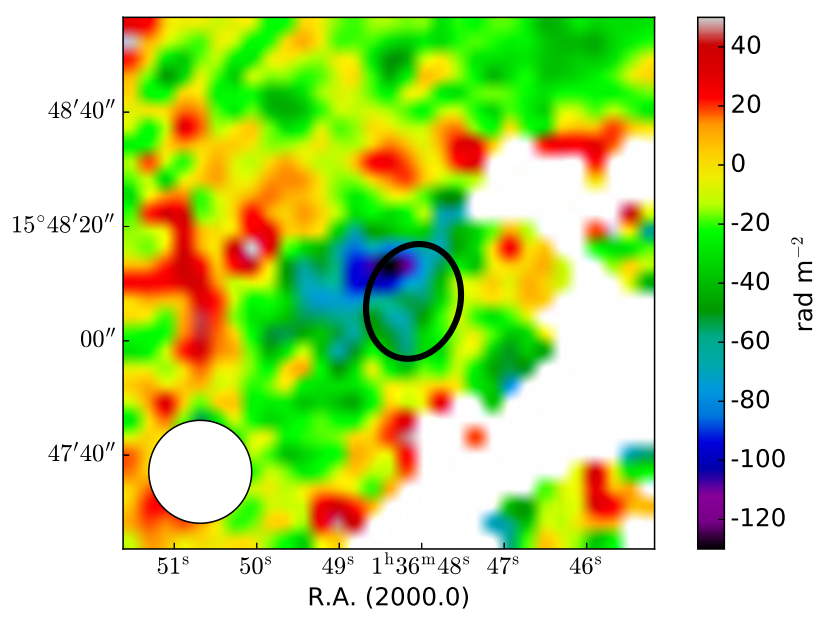

Fig. 23. Region of NGC 628 with a large Faraday depth coinciding with a known $\mathrm{H}_{\mathrm{I}}$ hole marked by an ellipse. The $18^{\prime \prime}$ beam is shown in the bottom left, filled in white.

from star formation episodes in the disk (Norman \& Ikeuchi 1988).

No other Faraday depth feature coincides with $\mathrm{H}_{\mathrm{I}}$ holes across the galaxy. The reason for this is deferred until the discussion later in the paper (Sect. 6.1).

\subsection{Pitch angles of the magnetic field}

To determine the intrinsic magnetic pitch angle, the observed $\boldsymbol{B}$ vector map taken from the RM synthesis cube and thus corrected for Faraday rotation is transformed into the galaxy's plane and the position angle of the local circumferential orientation is subtracted. The resulting map (Fig. 24) shows that the polarisation arms 2 and 3 extending from the central region in the south-east to the north-west have a relatively constant and large magnetic pitch angle, while the (broader) polarisation arm 1 extending from the central region in the north-west over east to the south shows strongly fluctuating pitch angles. In the southern part of arm 1, the pitch angle is smaller at the outer edge compared to the inner edge.

The magnetic field lines seem to generally follow the orientations of the spiral pattern seen in polarised intensity. A quantitative analysis needs measurement of the pitch angle of the spiral arm structures observed in polarised intensity. Spiral arms with constant pitch angles are linear structures in polar coordinates $[\ln (r), \phi]$ where $r$ is the radius and $\phi$ the azimuthal angle. Figure 25 shows the polar transform of the PI map retrieved from RM synthesis (Fig. 18).

Table 8 lists the spiral features that are marked in Fig. 25. The average morphological pitch angles follow from the slopes in the polar plot, while the average magnetic pitch angles are measured from Fig. 24. The remarkable result is that the magnetic pitch angles are systematically larger than the morphological pitch angles of the spiral arms. The average difference is about $23^{\circ}$ for arm 1 , about $12^{\circ}$ for arm 2 , and about $15^{\circ}$ for arm 3 . The morphological pitch angle varies much more along a spiral arm than the magnetic pitch angle; the magnetic pattern is smoother than the spiral arm pattern. A magnetic pitch angle that is systematically larger than the morphological pitch angle of the spiral arms was also found for the galaxies M 83 (Frick et al. 2016) and M 101 (Berkhuijsen et al. 2016). This finding is further discussed in Sect. 6.3.

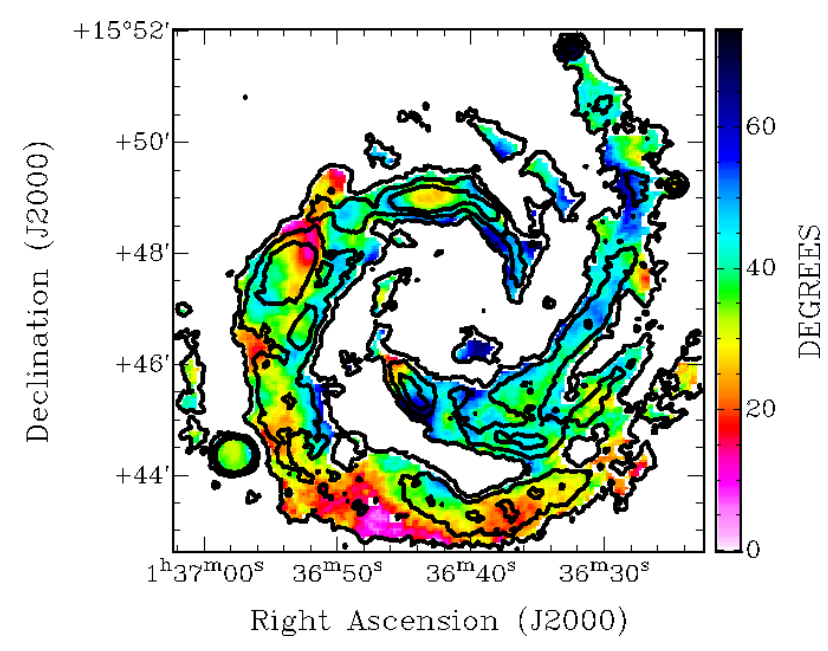

Fig. 24. Intrinsic pitch angles of the magnetic field in the plane of NGC 628 at $18^{\prime \prime}$ resolution. Contours show the polarised intensity at $5,10,15 \times \sigma_{\text {rms }}$.

\subsection{Faraday depth signatures of Parker loops and helical fields}

An azimuthal profile of Faraday depths was created by averaging the map along an annulus from a central point at $\operatorname{RA}(J 2000)=01^{\mathrm{h}} 36^{\mathrm{m}} 42^{\mathrm{s}} .4, \operatorname{Dec}(\mathrm{J} 2000)=+15^{\circ} 46^{\prime} 10^{\prime \prime}$ with a radius of $2.85^{\prime}$ and a width of $0.9^{\prime}$ which traces the eastern polarisation arm (polarised arm 1) from north to south (Fig. 26). Peaks are located at azimuthal angles of 31, 49, 67, 83, 101, 118, 143, 164,168 , and $175^{\circ}$. If we restrict the analysis to the range of azimuthal angles from $30^{\circ}$ to $130^{\circ}$, we are left with five regularly spaced peaks with an average separation of $17.5 \pm 0.5^{\circ}$, corresponding to a pattern wavelength of $35 \pm 1^{\circ}$ or $3.7 \pm 0.1 \mathrm{kpc}$.

The numerical simulations by Kim et al. (2002) predict a wavelength of the most unstable symmetric mode of the Parker instability of between $4 \pi h$ and $17 h$ where $h$ is the $\mathrm{H}_{\text {I gas scale }}$ height. The wavelength measured from our observations corresponds to a $\mathrm{H}$ i gas scale height of $108-147 \mathrm{pc}$. The average scale height for H I gas in NGC 628 is 490 pc (Bagetakos et al. 2011). This discrepancy is likely due to the fact that Kim et al. (2002) assumed $\beta=1$, where $\beta$ is the ratio of thermal to magnetic energy densities, whereas $\beta \ll 1$ was found in NGC 6946 and IC 342. Additionally, the calculated scale height could also be inaccurate due to assumptions made in Bagetakos et al. (2011) such as adopting a constant velocity dispersion for the gas.

Field loops are observed only in the eastern part of polarisation arm 1. Rodrigues et al. (2016) performed numerical simulations of Parker instabilities including cosmic rays that can exert a pressure comparable to thermal gas, turbulence and magnetic fields. They showed that the observed Faraday depth signature is a periodic pattern, with its peak magnitude and wavelength depending on the ratio of cosmic ray pressure to gas pressure (see Fig. 12 of that paper). When the cosmic ray pressure is dominant this Faraday depth signature can develop within $200 \mathrm{Myr}$, albeit, the peak magnitude and separation are small. When the gas pressure is dominant, the Faraday depth signature develops more slowly but the peak magnitude and wavelength are large. In the eastern part of polarisation arm 1, where the field loops are observed, significant amounts of gas and star formation are present. In other regions, such as the magnetic arms 2 and 3 where we do not observe an alternating Faraday depth signature, the cosmic ray pressure will be more comparable to the gas pressure which in turn produces smaller Faraday depth peak magnitudes 


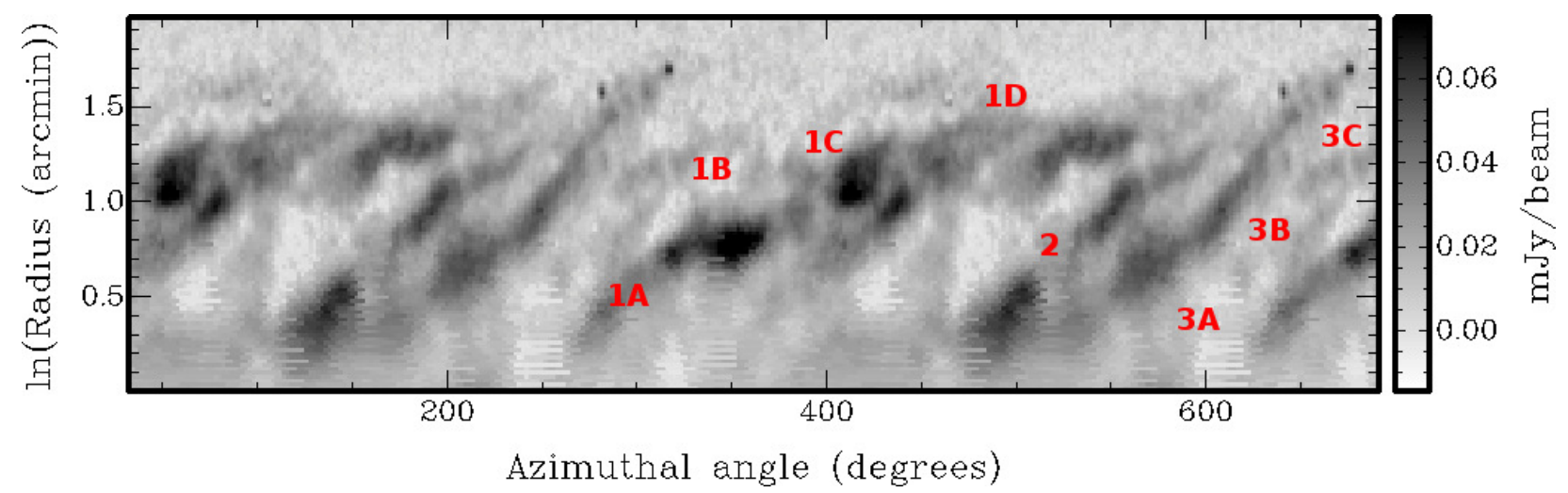

Fig. 25. Polarised intensity of NGC 628 at $18^{\prime \prime}$ in polar coordinates (azimuthal angle in degrees, measured counterclockwise from the north-eastern major axis in the galaxy plane, and $\mathrm{ln}$ of radius in arcminutes). The range of azimuthal angles is plotted twice for better visibility of the spiral arms. The recognised structures in the polarisation arms for which the structure and magnetic pitch angles are found (Table 8) are labelled in red.

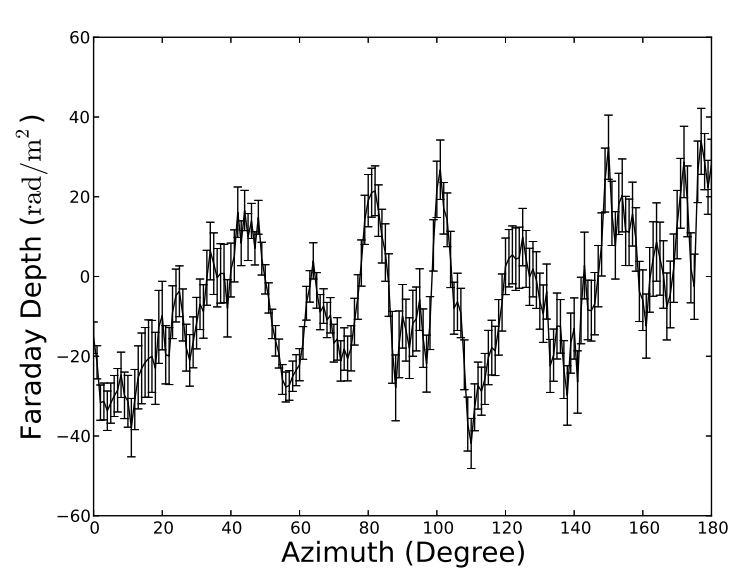

Fig. 26. Azimuthal profile of Faraday depth measured in sectors of a ring along the eastern part of polarisation arm 1 . North is at $0^{\circ}$, east at $90^{\circ}$ and south at $180^{\circ}$. The error bars give the error of the mean value in each sector, using the error map of Fig. 18 (right).

and pattern wavelengths, so much smaller in fact that we may not possess the Faraday depth and angular resolution needed to resolve these features.

Faraday depth signatures of Parker loops could exist throughout the galaxy but better Faraday depth and angular resolution as well as sensitivity are needed to reveal them. Further analysis and observations will be performed to further explore this issue.

Models by Hanasz et al. (2002) indicate that the Parker instability develops a wave-like deformation with a small amount of twisting at the base of the instability that occurs after $400 \mathrm{Myr}$ before magnetic reconnection begins and becomes a helical field at large heights (see Fig. 9 of their paper). Helical fields on scales of the typical turbulence length of $50-100 \mathrm{pc}$ can also be generated by the large-scale dynamo (e.g. Beck et al. 1996) from which larger loops may be formed by reconnection. Our resolution of $18^{\prime \prime}$ corresponds to about $640 \mathrm{pc}$.

A signature of helical fields on such scales would be a correlation between Faraday depth (Fig. 18, left) and intrinsic pitch angle (Fig. 24). We computed a pixel to pixel correlation for every fifth pixel between these two quantities at pixels where the polarisation signal-to-noise ratio is larger than 8 , and for each spiral arm individually. We found a weak anti-correlation $\left(\rho=-0.2\right.$ with a $p$-value of $\left.2.3 \times 10^{-6}\right)$ between the Faraday depth and the pitch angle in the eastern part of the polarisation
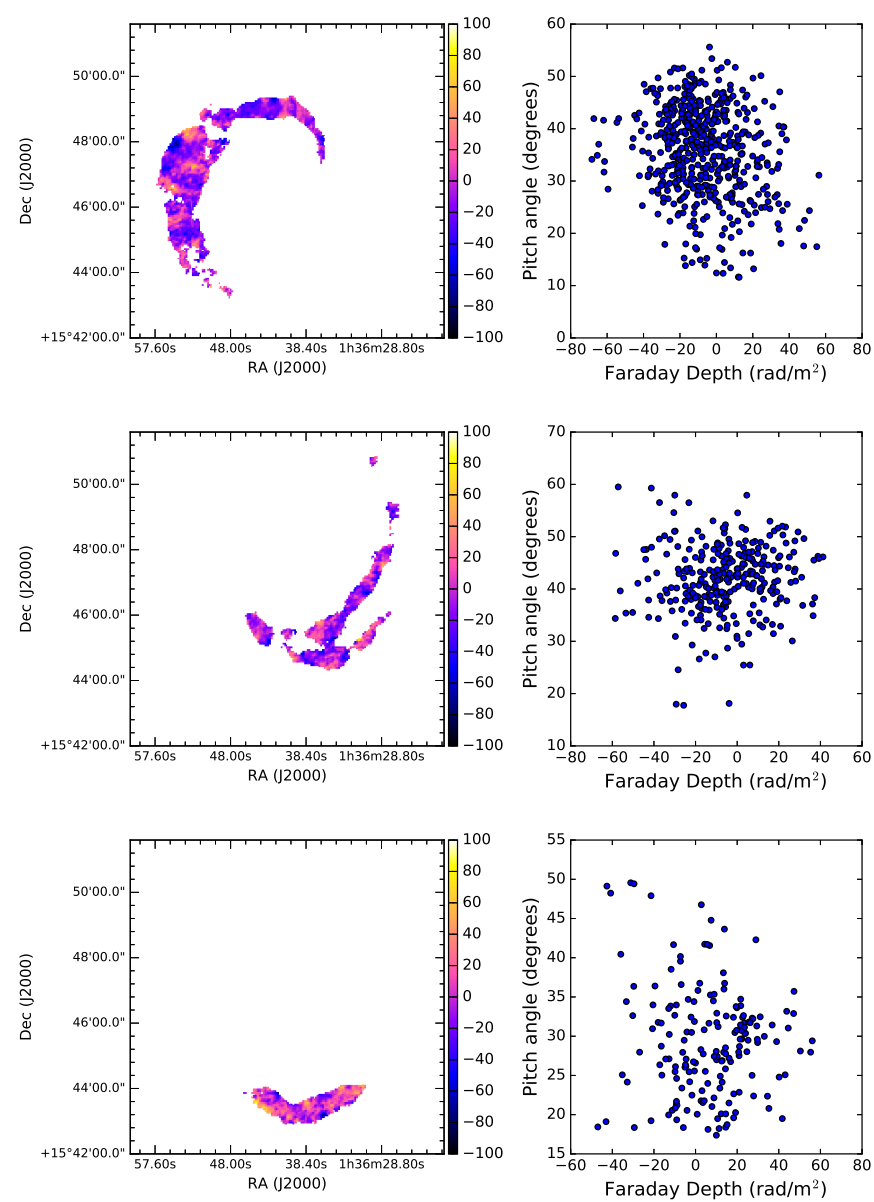

Fig. 27. A pixel to pixel correlation between Faraday depth and pitch angle (right) corresponding to each polarised arm shown in the colour maps of Faraday depth to the left, with the colour scale in $\mathrm{rad} \mathrm{m}^{-2}$.

arm 1 (Fig. 27), which is in conflict with the helical field interpretation (see Sect. 6.2 for a discussion).

No correlation whatsoever is seen for the southern part of the polarisation arm $1(\rho=-0.1)$ or for the polarisation arms 2 and $3(\rho=0.06)$ that do not show any regularly spaced peaks, unlike the eastern part of arm 1. Correlations between Faraday depth and total \& polarised intensity were also checked but no meaningful correlation could be seen in any of the arms. 


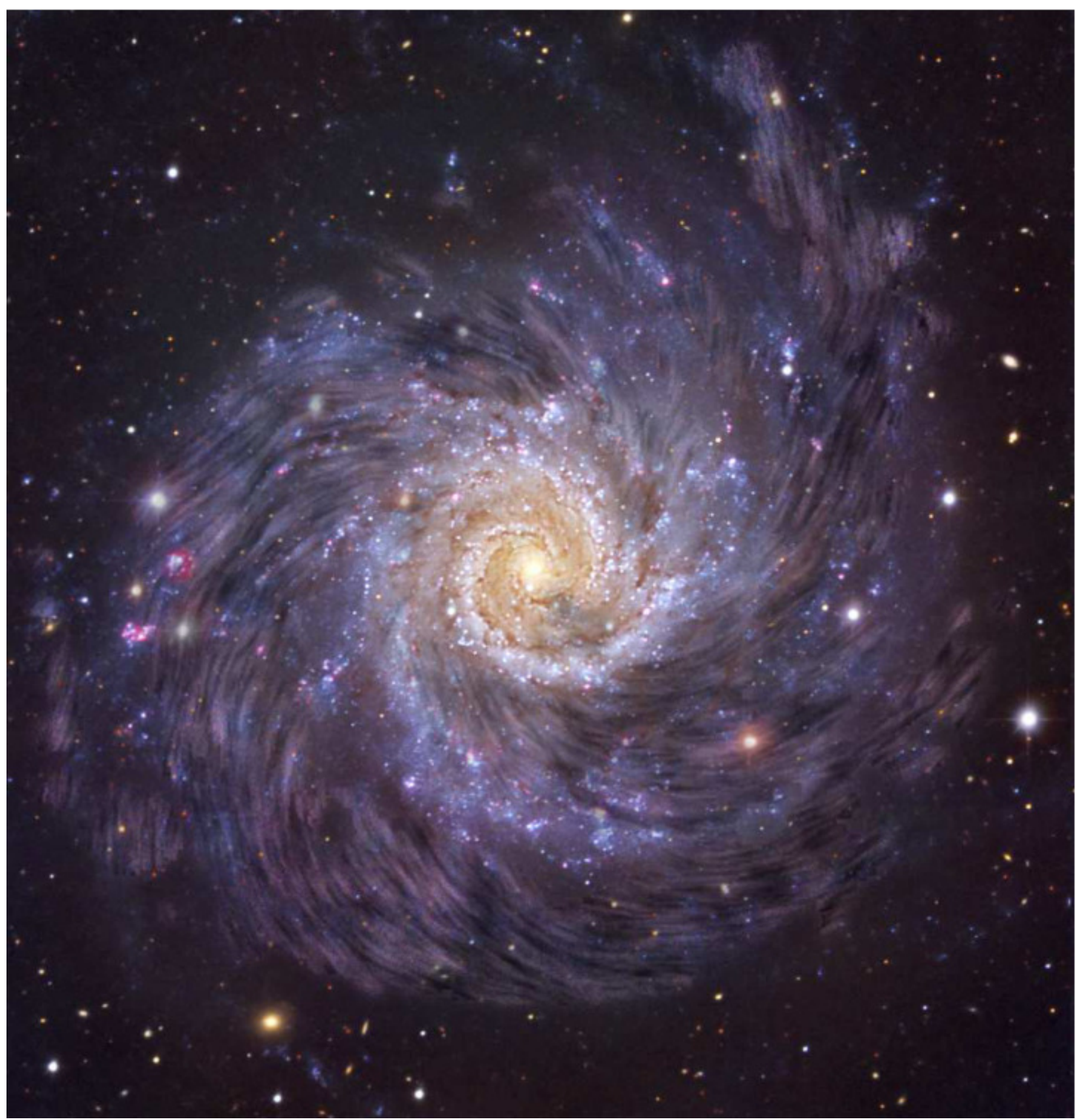

Fig. 28. Polarised intensity of NGC 628 with the magnetic fields illustrated as flow lines using the Line Integral Convolution method (Cabral \& Leedom 1993). The background image is an optical image plus $\mathrm{H} \alpha$ from the Calar Alto Observatory in Spain, created by Steve Mazlin and Vicent Peris (please see the acknowledgements for the full list of contributors).

\section{Discussion}

\subsection{Why does only one Faraday depth feature coincide with an H I hole?}

The co-location of a Faraday depth gradient with an $\mathrm{H}_{\text {i }}$ hole found in this work suggests that the discovery in NGC 6946 (Heald 2012) is not artificial. This is consistent with superbubbles being the main energy driver in the disk-halo interface. The Faraday depth observed in the Faraday depth gradient in
NGC 6946 (Heald 2012) ranges from 18.8 to $57.0 \mathrm{rad} \mathrm{m}^{-2}$, a range considerably smaller than the Faraday resolution of our observation. Possibly we do not possess the Faraday depth resolution to detect similar Faraday depth gradients. We may be observing the most extreme Faraday depth gradient in NGC 628 of about $110 \mathrm{rad} \mathrm{m}^{-2}$ (Fig. 7), more than twice compared to what is seen in NGC 6946.

There are several possible reasons why only one Faraday depth gradient coinciding with an $\mathrm{H}_{\mathrm{I}}$ hole is observed. Firstly, 
more than half (58 out of 102 ) of the $\mathrm{H}_{\mathrm{I}}$ holes in the disk of NGC 628 are located in regions devoid of $S$-band polarised emission due to more intense star formation, accompanied by an increased level of depolarisation. The age of the $\mathrm{H}_{\mathrm{I}}$ hole is also important and must be within a certain range, in that it must be old enough so that the magnetic field configuration is able to gain a significant vertical offset but young enough that the vertical shear has not destroyed the observational evidence of the Faraday depth gradient. Additionally, the azimuthal location is important in order to create an ideal geometric situation and thus produce an observable gradient. The small Faraday depolarisation effect (Fig. 20) indicates that polarimetry in $S$-band is probing the disk close to the midplane where strengths of the turbulent magnetic field and thermal electron densities are large. The magnetic field may not have had enough time to stabilise its configuration due to the turbulence near the midplane.

JVLA polarimetric observations in $L$-band (1-2 GHz) with its better Faraday depth resolution (about $90 \mathrm{rad} \mathrm{m}^{-2}$ ) are superior for finding smaller Faraday depth gradients. $L$-band observations would reveal polarisation at larger heights above the midplane (closer to the observer), a region where the magnetic field configuration is able to create an observable Faraday depth gradient. However, Mao et al. (2015) found no coincidence between $\mathrm{H}_{\mathrm{I}}$ holes and Faraday depth gradients in M 51 observed in $L$-band, so it remains to be seen if additional Faraday depth gradients can be detected in NGC 628 allowing a more comprehensive analysis of the field configuration in the disk-halo interface.

\subsection{Parker loops without helical fields?}

Faraday depth is generated by the magnetic field along the line of sight which is the vertical field in a galaxy with a small inclination like NGC 628. We observed a striking periodic pattern of alternating Faraday depths (see Sect. 5.8) from positive to negative Faraday depths (Fig. 26) along one of the spiral arms. The wavelength of this pattern corresponds to $3.7 \pm 0.1 \mathrm{kpc}$. This kind of feature can be described as a magnetic loop extending out of the galaxy's disk into its halo. Similar loops have been observed in IC 342 (Beck 2015) with a pattern wavelengths of about $4.4 \mathrm{kpc}$.

A regular pattern of Faraday depths could be a signature of large-scale helical fields around the spiral arm if the pitch angles of the field (Fig. 24), delineating the field component in the galaxy plane, reveal a periodicity with a similar separation. The weak anti-correlation between pitch angle and Faraday depth (Fig. 27) shows that the regular magnetic fields observed in NGC 628 are hardly of helical structure and hence are different from those observed in M 31 and IC 342. We conclude that the observed field deviations occur mostly in the vertical direction, in contrast to the models by Hanasz et al. (2002).

NGC 628 and IC 342 have similar Faraday depths, so that the vertical magnetic field strengths (and the electron densities) are similar. IC 342 has a SFR per surface area of about $0.016 M_{\odot} \mathrm{yr}^{-1} \mathrm{kpc}^{-2}$ in comparison to $0.0026 M_{\odot} \mathrm{yr}^{-1} \mathrm{kpc}^{-2}$ in NGC 628 (Calzetti et al. 2010). A higher SFR should result in a greater outflow speed into the halo. Additionally, the SFR across the disk in NGC 628 steadily decreased with time (see Sect. 4.3), thereby preventing a constant outflow in NGC 628.

The twisting of Parker loops is caused by two main factors, the rotational velocity of the galaxy and the horizontal component of the velocity vector due to gas flow along the magnetic field lines. NGC 628 and IC 342 have similar rotation curves (Kamphuis \& Briggs 1992; Sofue 1996), while IC 342 is expected to have a more continuous and stronger outflow into the halo compared to NGC 628 (Sect. 4.3). This has two consequences. Firstly, the polarised emission from NGC 628 traces mainly the disk. As the Parker loops are not twisted already near to the galaxy plane, the observed magnetic pitch angle is not affected. Secondly, a large halo like in IC 342 is more efficient in the inflation of Parker loops (Parker 1992) because helical fields extend to heights of several $\mathrm{kpc}$, similar to their pattern wavelengths.

If the regular pattern of Faraday depths is indeed caused by Parker loops, this should affect the dynamics of the ionised gas, which would be able to slide down the magnetic field loops with signatures in spectroscopic observations. A follow-up spectroscopic $\mathrm{H} \alpha$ observation along the regular pattern of Faraday depths (especially in regions bright in $\mathrm{H} \alpha$, see for example Fig. 13) would be beneficial to determine which effect the observed Parker loops have on the dynamics of the ionised gas.

\subsection{Magnetic pitch angles}

The maintenance of both radial and azimuthal components of the magnetic field, and hence a non-zero pitch angle, is an important prediction of dynamo theory (Beck et al. 1996), which is confirmed by many observations like in NGC 628. More crucially, the magnetic pitch angle is always larger than the morphological pitch angle of the spiral arms, as was also found for the galaxies M 83 (Frick et al. 2016) and M 101 (Berkhuijsen et al. 2016). This gives evidence for the action of a large-scale dynamo where the magnetic field is not coupled to the gas flow and obtains a significant radial component. Our NGC 628 data give further support to this model. The smoothness of the magnetic pitch angle is also consistent with dynamo action because the large-scale field is built up over several galactic rotations and is not strongly affected by local features in the spiral arms.

The dynamo models by Moss et al. $(2013,2015)$ assume that a large-scale ordered field is generated everywhere in the disk, while a small-scale dynamo injects turbulent fields only in the spiral arms. This gives polarisation arms between the gaseous arms at all radii but with pitch angles of the polarisation structures and pitch angles of the magnetic field lines that are significantly smaller than those of the gaseous arms. This is in contrast to the observations discussed in this paper. Applying the wavelet transform technique by Frick et al. (2016) to NGC 628 will allow a more detailed analysis which is essential for a deeper understanding of the interaction between spiral structure and magnetic fields.

The smoothness of the magnetic pitch angle is also worth discussing. As mentioned previously this smoothness is consistent with dynamo action. Moss et al. (2013) performed a 2D mean-field dynamo model in the "no-z" approximation adding injections of a small-scale magnetic field. These injections are the result of supernova shock fronts situated in the spiral arms and represent the effects of the dynamo at smaller scales. In particular, the model with a lower star formation rate ("model 77"), and hence less injection of turbulent field into the spiral arms, can produce a smooth magnetic pitch angle. While this model produces a broad polarisation arm that almost fills the inter-arm regions, the western arm of NGC 628 is not broad but shows very filamentary structure with the magnetic arm splitting into two (Fig. 14). Moss et al. (2013) pointed out that the field injection could decrease more slowly and smoothly away from the arms and that could produce a more filamentary structure. NGC 628 poses the interesting question what may occur if the injection of the small-scale magnetic field in the central region disappears. While this decrease in star formation in the central region has only occurred in the past $100 \mathrm{Myr}$, the timescale is probably not 
long enough to significantly change the dynamo. This calls for further developments of the dynamo models.

Direct evidence for a dynamo-generated large-scale field in the disk can be derived from the observation of a large-scale pattern of Faraday rotation measures or Faraday depths (e.g. Beck \& Wielebinski 2013). However, this method cannot be applied to NGC 628 due to its small inclination.

\section{Conclusions}

In this work we have presented new observations of NGC 628 with the JVLA at $S$-band (2-4 GHz) and Effelsberg 100-m telescope at $8.35 \mathrm{GHz}$ and $2.6 \mathrm{GHz}$. The wide bandwidth of the JVLA has enabled us to image NGC 628 with unprecedented sensitivity and resolution. The wide bandwidth also allowed us not only to perform RM synthesis but investigate the Faraday depolarisation across the band. Significant polarisation was observed across the disk especially strong in the interarm regions, similar to NGC 6946 and IC 342. Three main polarisation arms are evident but have different properties, with two arms filling the criterion for a magnetic arm. Until now NGC 628 has been relatively unexplored in radio continuum but with its extended $\mathrm{H}$ I disk and lack of active star formation in its central region has produced a wealth of interesting magnetic phenomena. We observe evidence for two drivers of magnetic turbulence in the disk-halo connection of NGC 628, namely, Parker instabilities and superbubbles.

The main findings of this work can be summarised as follows:

From our Effelsberg observations and data from literature we estimate an integrated spectral index for NGC 628 of $\alpha=$ $-0.79 \pm 0.06$ which is a normal value for spiral galaxies (Gioia et al. 1982). The spectrum is also consistent with Basu et al. (2015) that due to the clumpy nature of the ISM the galaxy-integrated spectrum shows no signs of CRE loss mechanisms and remains a power law over much of the radio continuum.

A smooth extension to the north of NGC 628 can be seen in total radio continuum coinciding with the extended $\mathrm{H}$ I disk as well as weak $\mathrm{H}$ II regions located in the outer disk. Additionally, there is evidence that one of the polarisation arms extends far into the extended disk. The polarised arms are seen to traverse along the optical interarm regions, as illustrated as flow lines using the Line Integral Convolution method (Cabral \& Leedom 1993) in Fig. 28.

We observe little radio emission and a high thermal fraction in the central region of the galaxy. The Initial Mass Function seems to be steeper than that in the spiral arms. While the central region hosts enough stars to ionise the gas that emits in $\mathrm{H} \alpha$, there are not enough very massive $\mathrm{O}$ stars that can become supernovae and produce CREs.

We computed magnetic field strengths using the nonthermal emission and find a mean magnetic field strength across the galaxy of $9 \mu \mathrm{G}$ out to a radius of $8 \mathrm{kpc}$. In addition, we observe a magnetic field strength of $12 \mu \mathrm{G}$ in the arm regions and $15 \mu \mathrm{G}$ in the largest star forming regions with $7 \mu \mathrm{G}$ in the extended disk beyond a radius of $8 \mathrm{kpc}$.

We observe a region in the north of the galaxy with intense polarised emission. The degree of field order is higher and the magnetic pitch angle is smaller compared to its immediate surroundings. We find this feature could be consistent with a barrel-shaped expansion taking place preferentially along the direction of the ordered magnetic field. Such a feature is not seen in other galaxies and possibly unique.

We find no clear indications of polarisation originating from the far side of the midplane, as observed in Braun et al. (2010). Better Faraday depth resolution is required in order to confirm this, e.g. with observations with the JVLA at $L$-band.

In the eastern part of polarisation arm 1 we observe a periodic pattern in Faraday depth with an average pattern wavelength of $3.7 \pm 0.1 \mathrm{kpc}$ which indicates Parker loops. A weak anticorrelation between Faraday depth and magnetic pitch angle suggests that these loops are vertical in nature with little helical twisting.

We observe one significant feature in Faraday depth which coincides with an $\mathrm{H}$ I hole. This signifies that the Faraday depth gradient seen in NGC 6946 by Heald (2012) is not coincidental. Such phenomena provide a mechanism for the dynamo process to expel magnetic fields in order to eliminate quenching.

We observe a magnetic pitch angle that is systematically larger than the morphological pitch angle which is also seen in M 83 (Frick et al. 2016) and M 101 (Berkhuijsen et al. 2016). This is evidence for the action of a large-scale dynamo where the magnetic field is not coupled to the gas flow and obtains a significant radial component. The smoothness of the magnetic pitch angle is also consistent with dynamo models, e.g. those by Moss et al. (2013, 2015).

Acknowledgements. D.D.M. acknowledges support from ERCStG 307215 (LODESTONE). This research was performed in the framework of the DFG Research Unit 1254 "Magnetisation of Interstellar and Intergalactic Media: The Prospects of Low-Frequency Radio Observations". Special thanks go to Aritra Basu for his help with this work and to Elly M. Berkhuijsen for carefully reading the paper. We also thank the anonymous referee whose helpful comments improved this paper. We thank CAHA, Descubre Foundation, DSA, Astronomical Observatory of the University of Valencia (OAUV), Vicent Peris (OAUV), José Luis Lamadrid (CEFCA), Jack Harvey (Star Shadow Remote Observatory, SSRO), Steve Mazlin (SSRO), Oriol Lehmkhul, Ivette Rodriguez, and Juan Conejero (PixInsight) for the permission to use their optical image (Fig. 28) of NGC 628.

\section{References}

Baars, J. W. M., Genzel, R., Pauliny-Toth, I. I. K., \& Witzel, A. 1977, A\&A, 61, 99

Bagetakos, I., Brinks, E., Walter, F., et al. 2011, AJ, 141, 23

Basu, A., Mitra, D., Wadadekar, Y., \& Ishwara-Chandra, C. H. 2012, MNRAS, 419, 1136

Basu, A., Beck, R., Schmidt, P., \& Roy, S. 2015, MNRAS, 449, 3879

Beck, R. 2007, A\&A, 470, 539

Beck, R. 2015, A\&A, 578, A93

Beck, R. 2016, A\&ARv, 24, 4

Beck, R., \& Krause, M. 2005, Astron. Nachr., 326, 414

Beck, R., \& Wielebinski, R. 2013, Magnetic Fields in Galaxies, eds. T. D. Oswalt, \& G. Gilmore (New York: Springer), 641

Beck, R., Brandenburg, A., Moss, D., Shukurov, A., \& Sokoloff, D. 1996, ARA\&A, 34, 155

Bell, A. R. 1978, MNRAS, 182, 443

Berkhuijsen, E. M., Urbanik, M., Beck, R., \& Han, J. L. 2016, A\&A, 588, A114 Boomsma, R., Oosterloo, T. A., Fraternali, F., van der Hulst, J. M., \& Sancisi, R. 2008, A\&A, 490, 555

Brandenburg, A., Moss, D., \& Shukurov, A. 1995, MNRAS, 276, 651

Braun, R., Heald, G., \& Beck, R. 2010, A\&A, 514, A42

Bregman, J. N. 1980, ApJ, 236, 577

Breitschwerdt, D., McKenzie, J. F., \& Völk, H. J. 1991, A\&A, 245, 79

Brentjens, M. A., \& de Bruyn, A. G. 2005, A\&A, 441, 1217

Burn, B. J. 1966, MNRAS, 133, 67

Cabral, B., \& Leedom, L. C. 1993, in Proc. 20th Annual Conference on Computer Graphics and Interactive Techniques, SIGGRAPH '93 (New York: ACM), 263

Calzetti, D., Wu, S.-Y., Hong, S., et al. 2010, ApJ, 714, 1256

Chamandy, L., Shukurov, A., \& Subramanian, K. 2015, MNRAS, 446, L6 
Chen, P. C., Cornett, R. H., Roberts, M. S., et al. 1992, ApJ, 395, L41

Chyży, K. T., \& Buta, R. J. 2008, ApJ, 677, L17

Clark, B. G. 1980, A\&A, 89, 377

Condon, J. 2015, ArXiv e-prints [arXiv: 1502 . 05616]

Condon, J. J. 1987, ApJS, 65, 485

Cornett, R. H., O’Connell, R. W., Greason, M. R., et al. 1994, ApJ, 426, 553

Cornwell, T. J. 2008, IEEE J. Selected Topics in Signal Processing, 2, 793

Dale, D. A., Cohen, S. A., Johnson, L. C., et al. 2009, ApJ, 703, 517

de Blok, W. J. G., Walter, F., Brinks, E., et al. 2008, AJ, 136, 2648

de Vaucouleurs, G., de Vaucouleurs, A., Corwin, Jr., H. G., et al. 1991, Third Reference Catalogue of Bright Galaxies. Volume I: Explanations and references. Volume II: Data for galaxies between $0^{\mathrm{h}}$ and $12^{\mathrm{h}}$. Volume III: Data for galaxies between $12^{\mathrm{h}}$ and $24^{\mathrm{h}}$.

Devereux, N. A., Price, R., Wells, L. A., \& Duric, N. 1994, AJ, 108, 1667

Dickinson, C., Davies, R. D., \& Davis, R. J. 2003, MNRAS, 341, 369

Emerson, D. T., \& Gräve, R. 1988, A\&A, 190, 353

Ferguson, A. M. N., Wyse, R. F. G., Gallagher, J. S., \& Hunter, D. A. 1998, ApJ, 506, L19

Frick, P., Stepanov, R., Beck, R., et al. 2016, A\&A, 585, A21

Gil de Paz, A., Boissier, S., Madore, B. F., et al. 2007, ApJS, 173, 185

Gioia, I. M., Gregorini, L., \& Klein, U. 1982, A\&A, 116, 164

Han, J. L., Beck, R., \& Berkhuijsen, E. M. 1998, A\&A, 335, 1117

Hanasz, M., Otmianowska-Mazur, K., \& Lesch, H. 2002, A\&A, 386, 347

Haslam, C. G. T. 1974, A\&AS, 15, 333

Heald, G. 2009, in IAU Symp. 259, eds. K. G. Strassmeier, A. G. Kosovichev, \& J. E. Beckman, 591

Heald, G., Braun, R., \& Edmonds, R. 2009, A\&A, 503, 409

Heald, G. H. 2012, ApJ, 754, L35

Heald, G. H., Rand, R. J., Benjamin, R. A., \& Bershady, M. A. 2006, ApJ, 647, 1018

Heald, G. H., Rand, R. J., Benjamin, R. A., \& Bershady, M. A. 2007, ApJ, 663 933

Ideguchi, S., Tashiro, Y., Akahori, T., Takahashi, K., \& Ryu, D. 2014, ApJ, 792, 51

Israel, F. P., \& Mahoney, M. J. 1990, ApJ, 352, 30

Kamphuis, J., \& Briggs, F. 1992, A\&A, 253, 335

Karachentsev, I. D., Karachentseva, V. E., Huchtmeier, W. K., \& Makarov, D. I. 2004, AJ, 127, 2031

Kennicutt, Jr., R. C., Armus, L., Bendo, G., et al. 2003, PASP, 115, 928

Kennicutt, R. C., Calzetti, D., Aniano, G., et al. 2011, PASP, 123, 1347

Kim, W.-T., Ostriker, E. C., \& Stone, J. M. 2002, ApJ, 581, 1080

Klein, U., Wielebinski, R., \& Beck, R. 1984, A\&A, 135, 213

Krause, M. 1993, in The Cosmic Dynamo, eds. F. Krause, K. H. Rädler, \& G. Rüdiger, IAU Symp., 157, 305

Kroupa, P. 2001, MNRAS, 322, 231

Kroupa, P. 2002, Science, 295, 82

Kroupa, P., \& Weidner, C. 2003, ApJ, 598, 1076

Krügel, E. 2003, The physics of interstellar dust (Bristol: The Institute of Physics)
Lada, C. J., \& Lada, E. A. 2003, ARA\&A, 41, 57

Lelièvre, M., \& Roy, J.-R. 2000, AJ, 120, 1306

Mao, S. A., Zweibel, E., Fletcher, A., Ott, J., \& Tabatabaei, F. 2015, ApJ, 800, 92

Marcum, P. M., O'Connell, R. W., Fanelli, M. N., et al. 2001, ApJS, 132, 129

McMullin, J. P., Waters, B., Schiebel, D., Young, W., \& Golap, K. 2007, in Astronomical Data Analysis Software and Systems XVI, eds. R. A. Shaw, F. Hill, \& D. J. Bell, ASP Conf. Ser., 376, 127

Moss, D., Sokoloff, D., Beck, R., \& Krause, M. 2010, A\&A, 512, A61

Moss, D., Beck, R., Sokoloff, D., et al. 2013, A\&A, 556, A147

Moss, D., Stepanov, R., Krause, M., Beck, R., \& Sokoloff, D. 2015, A\&A, 578, A94

Murphy, E. J., Helou, G., Kenney, J. D. P., Armus, L., \& Braun, R. 2008, ApJ, 678,828

Mulcahy, D. D., Horneffer, A., Beck, R., et al. 2014, A\&A, 568, A74

Mulcahy, D. D., Fletcher, A., Beck, R., Mitra, D., \& Scaife, A. M. M. 2016, A\&A, 592, A123

Norman, C. A., \& Ikeuchi, S. 1988, in The Outer Galaxy, eds. L. Blitz, \& F. J. Lockman, (Berlin: Springer Verlag), Lect. Notes Phys., 306, 155

Paladino, R., Murgia, M., \& Orrú, E. 2009, A\&A, 503, 747

Parker, E. N. 1992, ApJ, 401, 137

Perley, R. A., \& Butler, B. J. 2013a, ApJS, 204, 19

Perley, R. A., \& Butler, B. J. 2013b, ApJS, 206, 16

Pflamm-Altenburg, J., \& Kroupa, P. 2008, Nature, 455, 641

Pflamm-Altenburg, J., Weidner, C., \& Kroupa, P. 2007, ApJ, 671, 1550

Rampadarath, H., Morgan, J. S., Soria, R., et al. 2015, MNRAS, 452, 32

Rau, U., \& Cornwell, T. J. 2011, A\&A, 532, A71

Rodrigues, L. F. S., Sarson, G. R., Shukurov, A., Bushby, P. J., \& Fletcher, A. 2016, ApJ, 816, 2

Ruzmaikin, A., Sokolov, D., \& Shukurov, A. 1988, Nature, 336, 341

Shapiro, P. R., \& Field, G. B. 1976, ApJ, 205, 762

Shukurov, A., \& Berkhuijsen, E. M. 2003, MNRAS, 342, 496

Shukurov, A., Sokoloff, D., Subramanian, K., \& Brandenburg, A. 2006, A\&A, 448, L33

Sofue, Y. 1996, ApJ, 458, 120

Soida, M., Beck, R., Urbanik, M., \& Braine, J. 2002, A\&A, 394, 47

Sokoloff, D. D., Bykov, A. A., Shukurov, A., et al. 1998, MNRAS, 299, 189

Tabatabaei, F. S., Beck, R., Krügel, E., et al. 2007a, A\&A, 475, 133

Tabatabaei, F. S., Krause, M., \& Beck, R. 2007b, A\&A, 472, 785

Tabatabaei, F. S., Berkhuijsen, E. M., Frick, P., Beck, R., \& Schinnerer, E. 2013, A\&A, 557, A129

Valls-Gabaud, D. 1998, PASA, 15, 111

van Haarlem, M. P., Wise, M. W., Gunst, A. W., et al. 2013, A\&A, 556, A2

Vollmer, B., Soida, M., Beck, R., et al. 2007, A\&A, 464, L37

Walter, F., Brinks, E., de Blok, W. J. G., et al. 2008, AJ, 136, 2563

Weidner, C., \& Kroupa, P. 2005, ApJ, 625, 754

Weidner, C., \& Kroupa, P. 2006, MNRAS, 365, 1333

Weidner, C., \& Vink, J. S. 2010, A\&A, 524, A98

Weidner, C., Kroupa, P., \& Larsen, S. S. 2004, MNRAS, 350, 1503 